\title{
Updated properties of the old open cluster Melotte 66: Searching for multiple stellar populations ${ }^{\star}, \star \star$
}

\author{
Giovanni Carraro ${ }^{\star \star \star, 1}$, Gayandhi de $\mathrm{Silva}^{2}$, Lorenzo Monaco ${ }^{1}$, Antonino P. Milone ${ }^{3}$, and Renee Mateluna ${ }^{4}$ \\ 1 ESO, Alonso de Cordova 3107, 19001 Santiago de Chile, Chile \\ e-mail: [gcarraro, Imonaco] eeso.org \\ 2 Australian Astronomical Observatory, 105 Delhi Rd, NSW 2113 North Ryde, Australia \\ e-mail: gdesilva@aao.gov.au \\ 3 Research School of Astronomy and Astrophysics, Australian National University, Mt Stromlo Observatory, Cotter Rd, Weston, \\ ACT 2611, Australia \\ e-mail: antonino.milone@anu.edu.au \\ 4 Departamento de Astronomía, Universidad de Concepción, Casilla 169 Concepcion, Chile \\ e-mail: rmateluna@astro-udec.cl
}

Received 27 February 2014 / Accepted 17 April 2014

\section{ABSTRACT}

\begin{abstract}
Context. Multiple generations of stars are routinely encountered in globular clusters but no convincing evidence has been found in Galactic open clusters to date.

Aims. In this paper, we use new photometric and spectroscopic data to search for multiple stellar population signatures in the old, massive open cluster, Melotte 66. The cluster is known to have a red giant branch wide in color, which could be an indication of metallicity spread. Also the main sequence is wider than what is expected from photometric errors only. This evidence might be associated with either differential reddening or binaries. Both hypothesis have, however, to be evaluated in detail before recurring to the presence of multiple stellar populations.

Methods. New, high-quality, CCD UBVI photometry have been acquired to this aim with high-resolution spectroscopy of seven clump stars that are complemented with literature data; this doubles the number of clump star members of the cluster for which high-resolution spectroscopy is available. All this new material is carefully analyzed in search for any spectroscopic or photometric manifestation of multiple populations among the cluster stars.

Results. Our photometric study confirms that the width of the main sequence close to the turn off point is entirely accounted for by binary stars and differential reddening with no need to advocate more sofisticated scenarios, such as metallicity spread or multiple main sequences. By constructing synthetic color-magnitude diagrams, we infer that the binary fraction has to be as large as $30 \%$ and their mass ratio in the range 0.6-1.0. As a by-product of our simulations, we provide new estimates of the cluster fundamental parameters. We measure a reddening $E(B-V)=0.15 \pm 0.02$, and confirm the presence of a marginal differential reddening. The distance to the cluster is $4.7_{-0.1}^{+0.2} \mathrm{kpc}$ and the age is $3.4 \pm 0.3 \mathrm{Gyr}$, which is somewhat younger and better constrained than previous estimates.

Conclusions. Our detailed abundance analysis reveals that, overall, Melotte 66 looks like a typical object of the old thin disk population with no significant spread in any of the chemical species we could measure. Finally, we perform a photometric study of the blue straggler star population and argue that their number in Melotte 66 has been significantly overestimated in the past. The analysis of their spatial distribution supports the scenario that they are most probably primordial binaries.
\end{abstract}

Key words. stars: abundances - open clusters and associations: general - Galaxy: disk

\section{Introduction}

With the exception of Ruprecht 106 (Villanova et al. 2013) and possibly Terzan 8 (Carretta et al. 2014), all the Milky Way old globular clusters studied so far show either photometric or spectroscopic signatures of multiple stellar populations. The parameter driving the presence or absence of more than one population seems to be the total mass, and much work has currently

\footnotetext{
* Based on observations collected at Paranal Observatory under program 088.D-0045 and 076.D-0220, and at Las Campanas Observatory. $\star \star$ Full Table 2 is only available at the CDS via anonymous ftp to cdsarc.u-strasbg.fr (130.79.128.5) or via http://cdsarc.u-strasbg.fr/viz-bin/qcat?]/A+A/566/A39 $\star \star \star$ On leave from Dipartimento di Fisica e Astronomia, Universitá di Padova, Italy.
}

been done to study the lowest mass globulars. As stressed by Villanova et al. (2013), Terzan 7, Palomar 3, and NGC 1783 can be good candidates to look at.

An interesting and different perspective can be to consider Galactic open clusters - in particular those few old open clusters that are still massive enough - and search for a signature of multiple generations among their stars. Unfortunately, old massive, open clusters are extremely rare in the Milky Way: first, because open clusters are not very massive at birth, and second, because they loose quite some mass during their lifetime, mostly due to the tidal interaction with the Milky Way and the dense environment of the Milky Way disk (Friel 1995). The potential interest of old open clusters in the context of multiple stellar generations has been recognized for a while, but so far only two clusters have been investigated in details: NGC 6791 (Geisler et al. 2012) and 
Berkeley 39 (Bragaglia et al. 2012). Both clusters have current masses $\sim 10^{4} M_{\odot}$; NGC 6791 is probably somewhat more massive than Berkeley 39. In the case of Berkeley 39, no signature of multiple populations were found, while there seem to be two groups of stars in NGC 6791 having different Na abundance.

It is important, however, to state as clear as possible that masses are difficult to estimate for open clusters because of the significant field star contaminations and the large presence of binaries, which affects both photometric and kinematic mass measures (Friel 1995).

Therefore, one is often left with crude mass estimates, which are based mostly on the appearance of the color magnitude diagram (CMD) and the number of, for example, clump stars. A visual inspection at old open cluster older than, say, 5 Gyr, shows that we are left with maybe only three probably massive star clusters besides NGC 6791 and Berkeley 39. They are Trumpler 5, Collinder 26,1 and Melotte 66. No estimate of their mass is available, but a quick inspection of their CMD immediately shows that they harbor roughly the same number of clump stars as NGC 6791 and Berkeley 39, and therefore, their mass should be roughly of the same order. It seems to us, therefore, that it is urgent to look at these few clusters, and in this paper we are going to discuss new photometric and spectroscopic material for one of them: Melotte 66.

The plan of the paper is as follows. In Sect. 2, we summarize the literature information on Melotte 66 as completely as possible. Section 3 describes our photometric dataset, and provides details on observation, data reduction, and standardization. A star count analysis is then performed in Sect. 4. Section 5 deals with the study of Melotte 66 photometry, and the derivation of its fundamental parameters via the comparison with theoretical models. In Sect. 6, we describe the spectroscopic data, while we perform a detailed abundance analysis in Sect. 7. The blue straggler population in Melotte 66 is investigated in Sect. 8 and, finally, the conclusions of our work are drawn in Sect. 9.

\section{Melotte 66 in the literature}

Melotte 66 has been the subject of many studies over the years. It was recognized early on as a potentially old cluster by King (1964) and Eggen \& Stoy (1962) but studied in detail for the first time only ten years later by Hawarden (1976, 1978). In these two papers, Hawarden highlighted very clearly what makes Melotte 66 particularly interesting. He made use of UBV photometry which is mostly photographic, to derive a CMD that revealed the cluster turn off (TO) region for the first itme.

Based on this diagram, Hawarden drew the attention on (1) a rich and wide-in-color red giant branch (RGB); (2) the lack of a subgiant region; (3) a prominent gap in the upper main sequence; (4) the presence of a group of blue stragglers; (5) a conspicuous clump of core He- burning stars and, finally; (6) an anomalous low metallicity $([\mathrm{Fe} / \mathrm{H}] \sim-0.3)$, as inferred from the cluster ultraviolet excess index $\delta(U-B)_{0.6}$. He also provided estimates of the cluster absolute distance modulus (12.4 mag), reddening (0.17), and age ( 6-7 Gyr), emphasizing its extreme location below the Galactic plane $(\sim 750 \mathrm{pc})$.

The Hawarden findings boosted a wealth of investigations aimed at understanding the cluster peculiarities, especially the low metallicity and the color spread at fixed luminosity in the RGB. Hawarden (1978) added a few more photoelectric observations and concluded that differential reddening is not the culprit for the RGB width in a short contribution.

Anthony-Twarog et al. (1979) improved the BV photometry of the cluster and provided a CMD of higher quality than

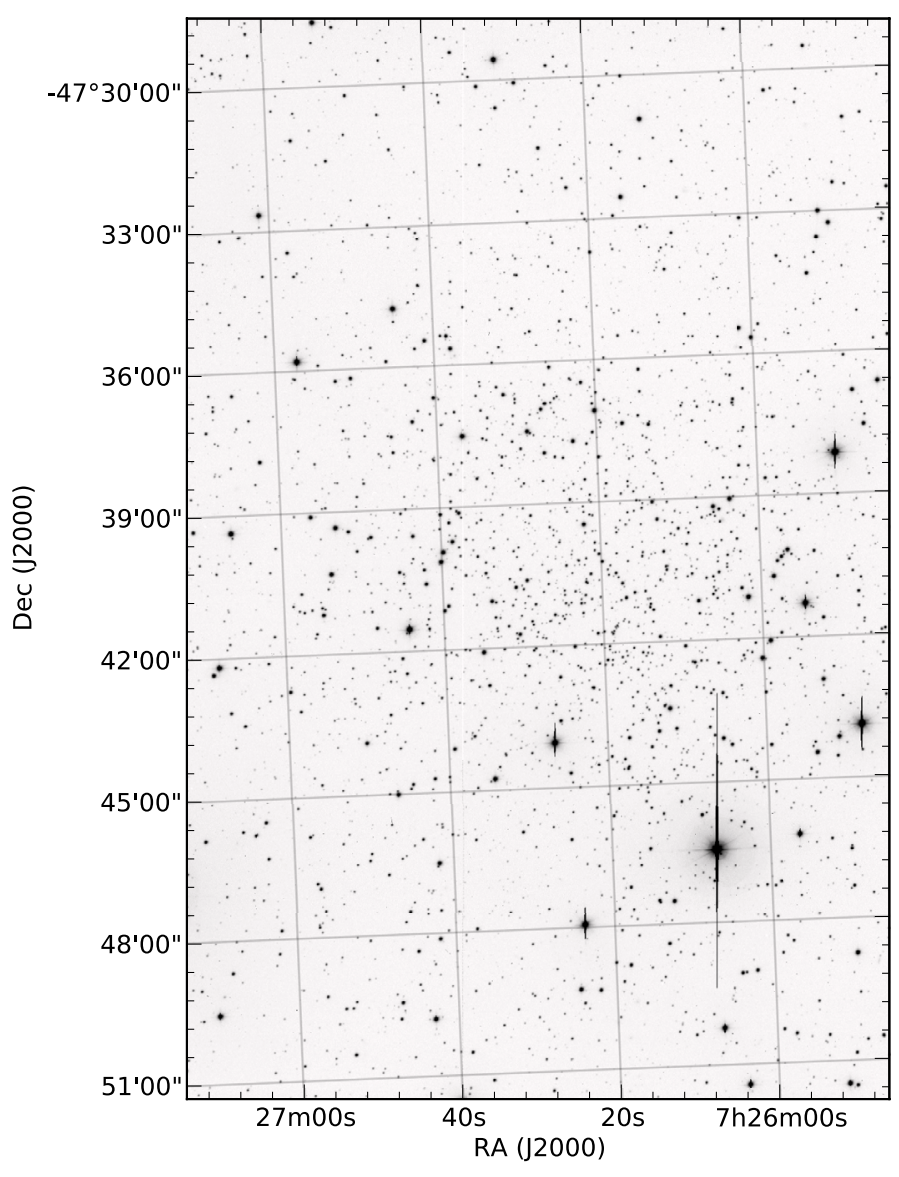

Fig. 1. An example of a CCD frame centered on Melotte 66. North is up, east to the left, and the field of view is $14.8 \times 22.8 \mathrm{arcmin}$. The image is in the $B$ filter, and the exposure was $1500 \mathrm{~s}$.

Hawarden, although with about the same magnitude limits. They also provided low resolution spectra and the star distribution in the CMD with theoretical isochrones for the first time. Their study reproduced all the features found by Hawarden, confirmed the metal abundance derived from his work on UBV photometry and from Dawson (1978) $\mathrm{DDO}^{1}$ photometry, and provided further support to Melotte 66's old age (6-7 Gyr), which about 1 Gyr older than NGC 188, and makes Melotte 66 the oldest open cluster know in those years. Finally, based on their $\mathrm{CN}$ strength, some blue RGB were suggested to be asymptotic giant branch (AGB) stars.

The first modern CCD study was conducted by Kaluzny \& Shara (1988) in the $B V$ pass-bands in a search for contact binaries. For the very first time, the main sequence (MS) of the cluster was revealed down to $V \sim 20$. The MS looked quite wide in perfect similarity to several Large Magellanic Cloud (LMC) clusters (Milone et al. 2009). No clear evidence of a significant binary population was, nonetheless, found.

A crucial step ahead in our understanding of these cluster properties come with the study by Anthony-Twarog et al. (1994). They presented CCD ubyH $\beta$ photometry down to $V \sim 20$. The MS was found to be much wider than expected from photometric errors, and the RGB width smaller than what expected from the MS. The subgiant branch was confirmed to be poorly populated. A metallicity spread implied by $m l^{2}$ index was found

\footnotetext{
David Dunlap Observatory photometric system.

$m 1=(v-b)-(b-y)$ is an index in the Stromgren system sensitive to metallicity (Crawford 1958).
} 


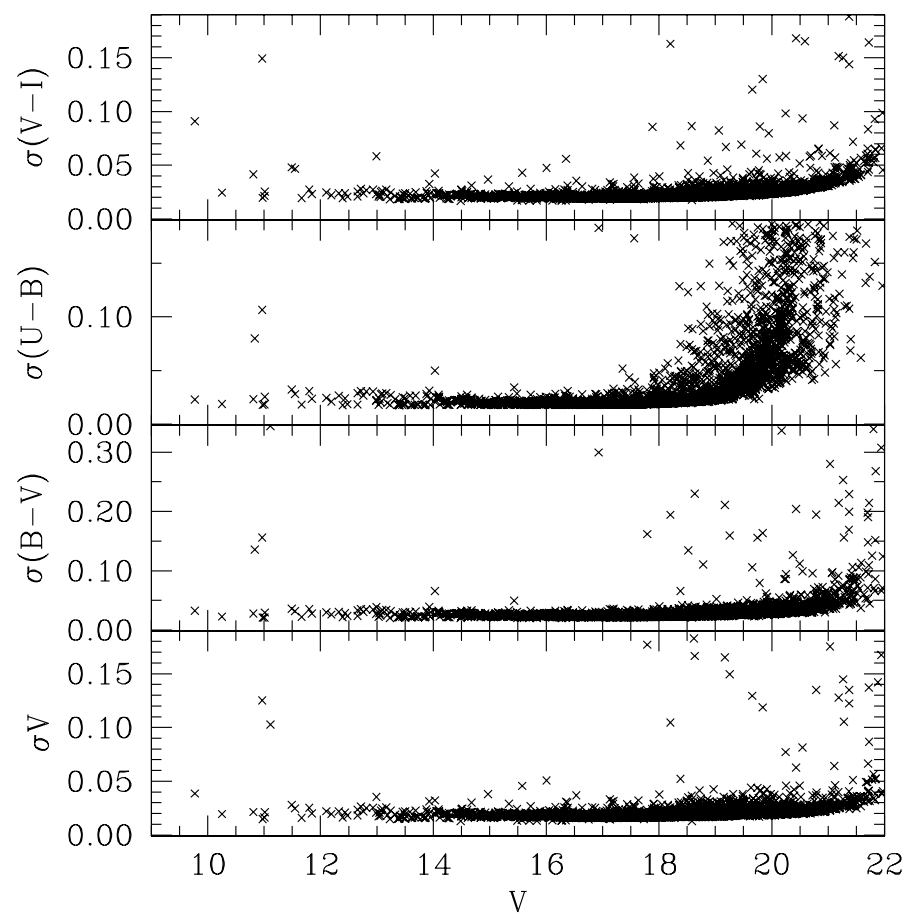

Fig. 2. Trend of global photometric errors in magnitude and colors as a function of $V$ magnitude. See text for details.

among RGB stars. Differential reddening across the cluster surface was excluded as the cause for the color spread in the CMD. The metallicity variation was also deemed to be not enough to account for the MS broadness, while star-to-star CN variation was conceived to be the most viable explanation for the color variation among RGB stars.

The photometric study that followed (Kassis et al. 1997; Zloczewski et al. 2007) provided very deep CCD photometry, extending the color coverage to the $I$ band and revealed a clear binary sequence parallel to the star cluster MS for the first time. Zloczeskwi et al. also constructed a reddening map, showing that reddening variations are significant across the cluster surface, at odds with Anthony-Twarog et al. (1994) study.

Finally, important pieces of information come from the spectroscopic studies carried out in the meanwhile. Friel \& Janes (1993) and Friel et al. (2002) first obtained medium resolution spectra of four giants in Melotte 66 and measured $[\mathrm{Fe} / \mathrm{H}]=$ $-0.51 \pm 0.11$, confirming that the cluster is indeed one of the most metal poor old open clusters in the Milky Way but does not show any significant spread in abundance.

Furthermore, two studies presented high resolution spectroscopy: Gratton \& Contarini (1994) and Sestito et al. (2008). The former obtained spectra of two giant stars, and concluded that $[\mathrm{Fe} / \mathrm{H}]=-0.38 \pm 0.15$, while the latter, a more detailed study, derived $[\mathrm{Fe} / \mathrm{H}]=-0.33 \pm 0.03$ from seven giants. This figure is very similar to Gratton \& Contarini (1994) and confirms that no spread in metallicity was detected among Mel 66 giants.

\section{Observations and data reduction: photometry}

The star cluster Melotte 66 was observed at Las Campanas Observatory (LCO) on the nights from January 3 to January 7, 2011, as illustrated in Table 1, which summarizes useful details of the observations, like filter coverage, airmass range, exposure time, and sequences. We used the SITe\#3 CCD detector
Table 1. $U B V I$ photometric observations of Melotte 66 and the standard star field SA 98.

\begin{tabular}{lcccc}
\hline \hline Date & Field & Filter & Exposures (s) & Airmass (X) \\
\hline Jan. 03, 2011 Mel 66 & $U$ & $60,3 \times 300,1500$ & $1.07-1.12$ \\
\hline Jan. 04, 2011 Mel 66 & $U$ & 1500 & 1.13 \\
& $B$ & $3 \times 45,3 \times 300,1500$ & $1.06-1.13$ \\
\hline Jan. 05, 2011 Mel 66 & $B$ & 1500 & 1.13 \\
& $V$ & $30,3 \times 300,2 \times 1200$ & $1.07-1.13$ \\
& $I$ & 30 & 1.06 \\
& SA 98 & $U$ & $4 \times 240$ & $1.17-1.67$ \\
& $B$ & $4 \times 120$ & $1.18-1.55$ \\
& $V$ & $4 \times 60$ & $1.18-1.52$ \\
& $I$ & $4 \times 60$ & $1.20-1.48$ \\
\hline Jan. 06, 2011 Mel 66 & $U$ & $5 \times 60,3 \times 300,2 \times 1500$ & $1.05-1.14$ \\
& $B$ & $2 \times 45,300$ & $1.10-1.13$ \\
& $V$ & $2 \times 30,300$ & $1.09-1.13$ \\
& $I$ & $3 \times 30,4 \times 300,2 \times 1000$ & $1.08-1.30$ \\
& SA 98 & $U$ & $4 \times 240$ & $1.18-1.84$ \\
& $B$ & $4 \times 120$ & $1.19-1.76$ \\
& $V$ & $4 \times 60$ & $1.20-1.70$ \\
& $I$ & $4 \times 60$ & $1.21-1.64$ \\
\hline Jan. 07, 2011 Mel 66 & $U$ & 1500 & 1.42 \\
& $B$ & $3 \times 45,3 \times 300,2 \times 1500$ & $1.15-1.30$ \\
& $V$ & $2 \times 30,3 \times 300,2 \times 1200$ & $1.05-1.10$ \\
& $I$ & $3 \times 30,3 \times 300,2 \times 1000$ & $1.06-1.10$ \\
\hline
\end{tabular}

onboard the Swope $1.0 \mathrm{~m}$ telescope $\mathrm{e}^{3}$. With a pixel scale of $0.435 \mathrm{arcsec} / \mathrm{pixel}$, this CCD allows to cover $14.8 \times 22.8 \mathrm{arcmin}^{2}$ on sky. The nights for which we observed standard stars were photometric with a seeing range from 0.8 to 1.5 arcsec. The field we covered is shown in Fig. 1, where a bias- and flat-fieldcorrected image in the $B$ band (1500 s) is shown.

We took a grand total of 68 images with a small jitter pattern, and eventually the montage frame covered an area of $16.4 \times 22.8 \operatorname{arcmin}^{2}$ on sky.

To determine the transformation from our instrumental system to the standard Johnson-Kron-Cousins system and to correct for extinction, we observed stars in Landolt's areas SA 98 (Landolt 1992), that contains many stars with good absolute photometry and wide color range. The field was observed multiple times with different air-masses ranging from $\sim 1.05$ to $\sim 1.9$ and covering quite a large color range $-0.3 \leq(B-V) \leq 1.7$ mag. We secured night-dependent calibrations (on January 05 and 06), which we then merged, after checking for stability.

\subsection{Basic photometric reduction}

Basic calibration of the CCD frames was done using IRAF package CCDRED. For this purpose, zero exposure frames and twilight sky flats were taken every night. All the frames were pre-reduced by applying trimming, bias and flat-field correction. Before flat-fielding, all frame were corrected for linearity, following the recipe discussed in Hamuy et al. (2006).

Photometry was then performed using the IRAF DAOPHOT/ ALLFRAME and PHOTCAL packages. Instrumental magnitudes were extracted following the point spread function (PSF)

\footnotetext{
3 http://wWW.1co.cl/telescopes-information/ henrietta-swope/

4 IRAF is distributed by the National Optical Astronomy Observatory, which is operated by the Association of Universities for Research in Astronomy, Inc., under cooperative agreement with the National Science Foundation.
} 


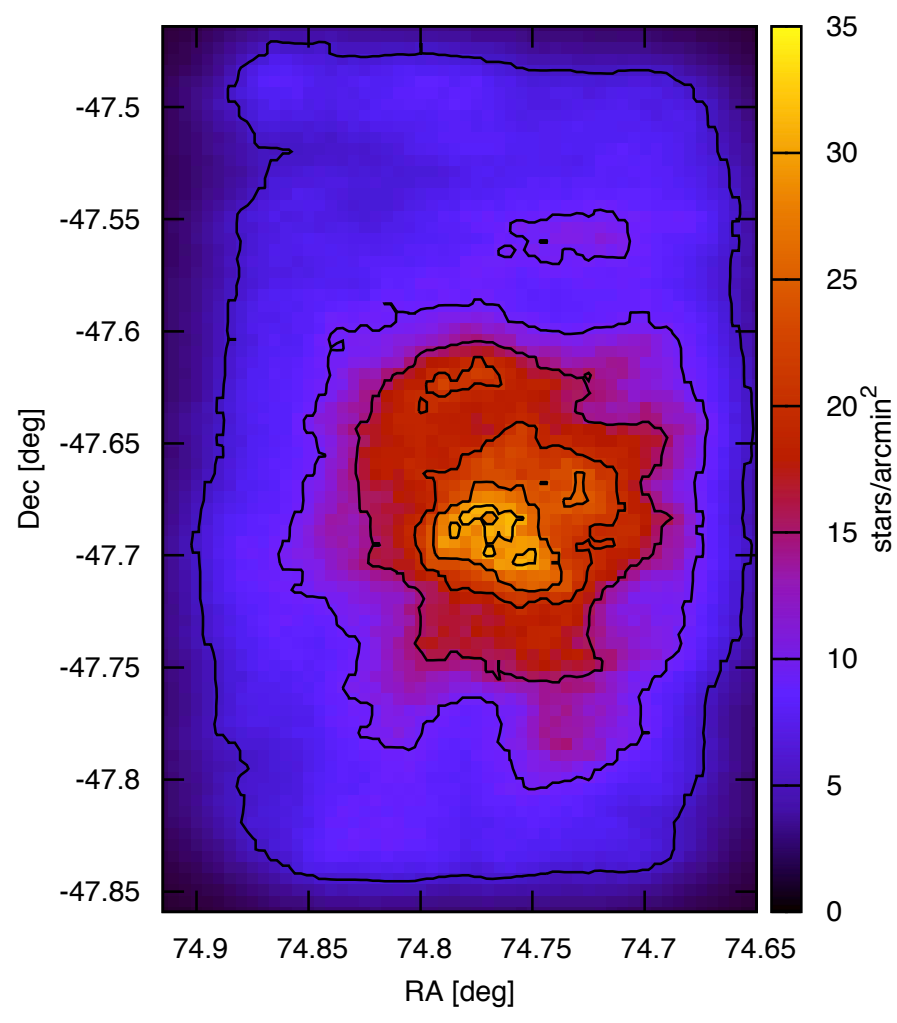

Fig. 3. Density contour map for Melotte 66 field. North is up, east to the left, and the field corresponds to $16.4 \times 22.8$ squared arcmin on sky. On the $X$-axis, $\mathrm{RA} \times \cos (\delta)$ is shown to keep the same scale as in Fig. 1 .

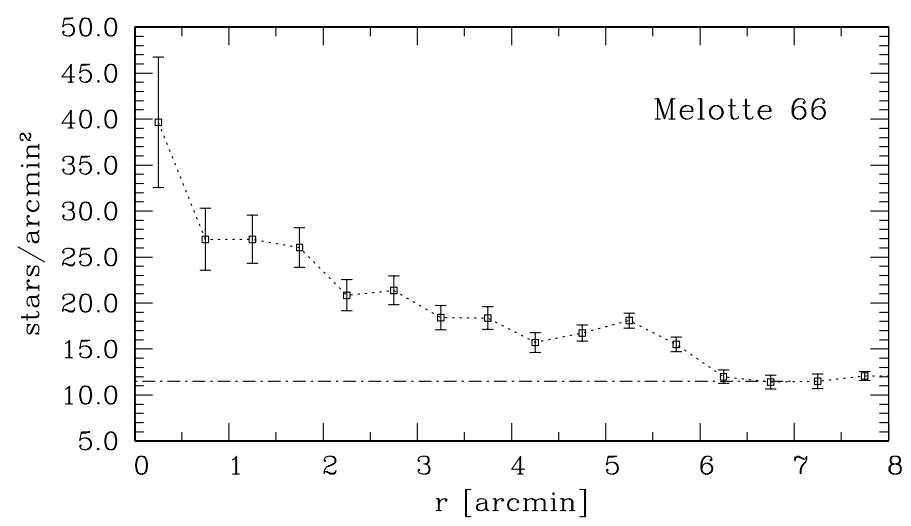

Fig. 4. Radial surface density profile. To define concentric rings, we used the nominal cluster center.

method (Stetson 1987). A quadratic, spatially variable master PSF (PENNY function) was adopted, because of the large field of view of the detector. Aperture corrections were then determined, making aperture photometry for a suitable number (typically 15 to 20 ) of bright, isolated, stars in the field. These corrections were found to vary from 0.160 to $0.290 \mathrm{mag}$, depending on the filter. The PSF photometry was finally aperture corrected filter-by-filter.

\subsection{Photometric calibration}

After removing problematic stars, and stars having only a few observations in Landolt's (1992) catalog, our photometric solution for the run was extracted by combining measures from both nights after checking if they were stable and similar. This yields a grand total of 89 measurements per filter and turned out to be:

$$
\begin{aligned}
& U=u+(4.921 \pm 0.012)+(0.47 \pm 0.01) \times X+(0.079 \pm \\
& 0.014) \times(U-B) \\
& B=b+(3.289 \pm 0.010)+(0.26 \pm 0.01) \times X+(0.074 \pm \\
& 0.009) \times(B-V) \\
& V=v+(3.193 \pm 0.007)+(0.16 \pm 0.01) \times X-(0.059 \pm \\
& 0.007) \times(B-V) \\
& I=i+(3.505 \pm 0.009)+(0.08 \pm 0.01) \times X+(0.052 \pm \\
& 0.006) \times(V-I)
\end{aligned}
$$

where $X$ indicates the airmass.

The final rms of the fitting in this case was 0.025, 0.018, 0.010, and 0.010 in $U, B, V$ and $I$, respectively.

Global photometric errors were derived using the scheme developed by Patat \& Carraro (2001, Appendix A1), which takes the errors resulting from the PSF fitting procedure (i.e., from ALLSTAR) and the calibration errors (corresponding to the zero point, color terms, and extinction errors) using errors' propagation into account. In Fig. 2, we present these global photometric errors in $V,(B-V),(U-B)$, and $(V-I)$ plotted as a function of $V$ magnitude. Quick inspection shows that stars brighter than $V \approx 20$ mag have errors lower than $\sim 0.05 \mathrm{mag}$ in both magnitude and the $(B-V)$ and $(V-I)$ colors. Larger errors, as expected, are seen in $(U-B)$.

The final catalog contains 3474 UBVI and 15752 VI entries.

\subsection{Completeness and astrometry}

Completeness corrections were determined by running artificial star experiments on the data. Figure 1 clearly shows that Melotte 66 does not suffer from serious crowding, and therefore, completeness has been evaluated over the whole cluster area. Basically, we created several artificial images by adding artificial stars to the original frames, on a frame-by-frame basis. These stars were added at random positions and had the same color and luminosity distribution of the true sample. To avoid generating overcrowding we added up to $20 \%$ of the original number of stars in each experiment. Depending on the frame, between 100 and 500 stars were added. In this way, we have estimated that the completeness level of our photometry is better than $90 \%$ down to $V \approx 20.5$ (see Table 3 ).

The optical catalog was then cross-correlated with 2MASS, which resulted in a final catalog that includes $U B V I$ and $J H K_{\mathrm{s}}$ magnitudes. As a by-product, pixel (i.e., detector) coordinates were converted to RA and Dec for J2000.0 equinox, thus providing 2MASS-based astrometry which is useful for spectroscopic follow-up. An excerpt of the optical photometric table used in this investigation is illustrated in Table 2.

\subsection{Comparison with previous photometry}

We compared our VI photometry with Kassis et al. (1991), as done by Zloczewski et al. (2007). From the 2303 common stars we obtain

$\Delta V=0.00 \pm 0.04$, and

$\Delta(V-I)=0.02 \pm 0.05$

in the sense of subtracting our photometry from the values determined Kassis et al. (1997). This implies that our VI photometry 
Table 2. An excerpt of the optical photometric table that includes clump with high-resolution spectroscopy.

\begin{tabular}{ccccccccccccc}
\hline \hline ID & Kassis et al. & $\begin{array}{c}\mathrm{RA}(2000.0) \\
\mathrm{deg}\end{array}$ & $\begin{array}{c}\mathrm{Dec}(2000.0) \\
\mathrm{deg}\end{array}$ & $V$ & $\sigma_{V}$ & $(U-B)$ & $\sigma_{(U-B)}$ & $(B-V)$ & $\sigma_{(B-V)}(V-I)$ & $\sigma_{(V-I)}$ \\
\hline 597 & 385 & 111.703333 & -47.65939 & 14.278 & 0.020 & 0.802 & 0.022 & 1.127 & 0.028 & 1.181 & 0.023 \\
776 & 603 & 111.679583 & -47.61947 & 14.378 & 0.020 & 0.810 & 0.022 & 1.137 & 0.028 & 1.142 & 0.022 \\
1521 & 1419 & 111.611250 & -47.63216 & 14.432 & 0.020 & 0.747 & 0.022 & 1.110 & 0.028 & 1.089 & 0.022 \\
2099 & 1953 & 111.572083 & -47.73336 & 14.551 & 0.021 & 0.735 & 0.022 & 1.118 & 0.028 & 1.167 & 0.023 \\
2209 & 2155 & 111.556250 & -47.62350 & 14.454 & 0.020 & 0.768 & 0.022 & 1.105 & 0.028 & 1.108 & 0.022 \\
2291 & 2187 & 111.554167 & -47.69819 & 14.553 & 0.020 & 0.746 & 0.022 & 1.107 & 0.028 & 1.147 & 0.023 \\
2803 & 2771 & 111.510417 & -47.68219 & 14.568 & 0.020 & 0.765 & 0.022 & 1.117 & 0.028 & 1.162 & 0.023 \\
1202 & 1000 & 111.644045 & -47.71306 & 14.640 & 0.021 & 0.757 & 0.022 & 1.151 & 0.028 & 1.182 & 0.023 \\
1734 & 1580 & 111.598675 & -47.70005 & 14.683 & 0.021 & 0.762 & 0.022 & 1.140 & 0.028 & 1.240 & 0.023 \\
2980 & 2945 & 111.491345 & -47.67310 & 14.495 & 0.020 & 0.855 & 0.023 & 1.139 & 0.028 & 1.147 & 0.022 \\
1919 & 1805 & 111.582833 & -47.67103 & 14.665 & 0.021 & 0.840 & 0.023 & 1.205 & 0.029 & 1.195 & 0.023 \\
\hline
\end{tabular}

Notes. The full version is available at the CDS. ID indicates the numbering. The last four stars have spectra taken from Sestito et al. (2008).

Table 3. Completeness study as a function of the filter.

\begin{tabular}{rrrrr}
\hline \hline$\Delta$ Mag & $U$ & $B$ & $V$ & $I$ \\
\hline $12-13$ & $100 \%$ & $100 \%$ & $100 \%$ & $100 \%$ \\
$13-14$ & $100 \%$ & $100 \%$ & $100 \%$ & $100 \%$ \\
$14-15$ & $100 \%$ & $100 \%$ & $100 \%$ & $100 \%$ \\
$15-16$ & $100 \%$ & $100 \%$ & $100 \%$ & $100 \%$ \\
$16-17$ & $100 \%$ & $100 \%$ & $100 \%$ & $100 \%$ \\
$17-18$ & $100 \%$ & $100 \%$ & $100 \%$ & $100 \%$ \\
$18-19$ & $100 \%$ & $100 \%$ & $100 \%$ & $100 \%$ \\
$19-20$ & $91 \%$ & $95 \%$ & $100 \%$ & $100 \%$ \\
$20-21$ & $72 \%$ & $80 \%$ & $95 \%$ & $96 \%$ \\
$21-22$ & $48 \&$ & $53 \%$ & $67 \%$ & $88 \%$ \\
\hline
\end{tabular}

is basically in the same system as Kassis et al. (1997) and Zloczewski et al. (2007).

The comparison in $U B V$ has been done against Zloczewski et al. (2007) with the only difference that the star in common having $(U-B)$ drops to 870 because of the lower sensitivity of these filters, which are mostly caused by the smaller amount of exposures. We find

$\Delta(B-V)=-0.04 \pm 0.03$, and

$\Delta(U-B)=0.06 \pm 0.04$.

We conclude that the two datasets agree fairly well also in $U B$, and therefore the two photometries are in the same system.

\section{Star counts and cluster size}

To be able to quantify the amount of field star contamination, we performed star counts to identify the cluster center and measure its size. To achieve this, we derived density contour maps using an array and calculated the density inside each grid step by a kernel estimate.

A quick glance at Fig. 1 shows that Melotte 66 is far from being a symmetric object. This is also visible in Fig. 3, which lends further support to the appearance of Fig. 1. The cluster is elongated in the direction NE to SW, and the highest peak does not represent the center of an uniform star distribution. The largest peak is located at RA $=111.57, \mathrm{Dec}=-47.71$, while the nominal center of the cluster is clearly displaced to the northeast direction, at: $\mathrm{RA}=111^{\circ} .60, \mathrm{Dec}=-47^{\circ} .66$. One can argue that the most probable reason for such an occurrence is the tidal interaction with the Milky Way. However, we do not have kinematic information, but only the cluster radial velocity. Highquality proper motions are still missing, and they would be very welcome to derive the cluster motion direction and see if this coincides with the direction of the apparent cluster elongation.

To isolate probable cluster members, which are this stars assumed to lie within the cluster boundaries, we derive the cluster radial surface density profile, which is shown in Fig. 4. This has been computed by drawing concentric rings centered on the nominal cluster center. This is motivated by the fact that the cluster halo still looks almost circular while the densest central regions look distorted. Star counts level off at $\sim 6$ arcmin from the cluster nominal center, confirming early findings by Hogg (1965) that the cluster diameter is around 13 arcmin. The mean density in the field surrounding the cluster is $10 \mathrm{stars} / \operatorname{arcmin}^{2}$ (see also Fig. 3), and our survey covers the whole cluster area. Our estimate of for the radial extent of Melotte 66 is smaller than Zlocewski et al. (2007). This is most probably because their star counts beyond 6 arcmin from the cluster center are not properl area-corrected (concentric rings are not complete anymore), thus producing artificial over densities. As a consequence, we will adopt a value of 6 arcmin for cluster radius ion the following. We will refer to to the area of the circle with a radius of 6 arcmin as the cluster area, while the area outside 6 arcmin from the cluster center is referred to as the offset field.

\section{Photometric diagrams}

The CMDs for all the stars in the observed field are shown in Fig. 4 for three different color combinations: $V / B-V, V / V-I$, and $V / U-B$ from the left to the right. All the features previously outlined by Anthony-Twarog et al. (1994) are visible in these diagrams. Since our field of view is larger, the field star contamination is more important and precludes a clear identification of all the CMD features. We can recognize a prominent MS that extends from $V \sim 16.5$ down to 20.5. This last limit does not depend on completeness or photometric depth but is only set by the constraint to have plotted all the stars having $U B V I$ measures.

While the RGB and the clump (at about $V \sim 14.5$ ) are well delineated, the subgiant branch is clearly blurred and confused by field stars. The field star MS crosses the subgiant branch and continues up to $V \sim 13.0$.

The blue straggler stars sequence is also well defined at $V \sim$ 16.0, $(B-V) \sim \leq 0.3$. Finally, on the right side of the MS, a conspicuous binary sequence is present, as previously outlined by Zlocewski et al. (2007). 


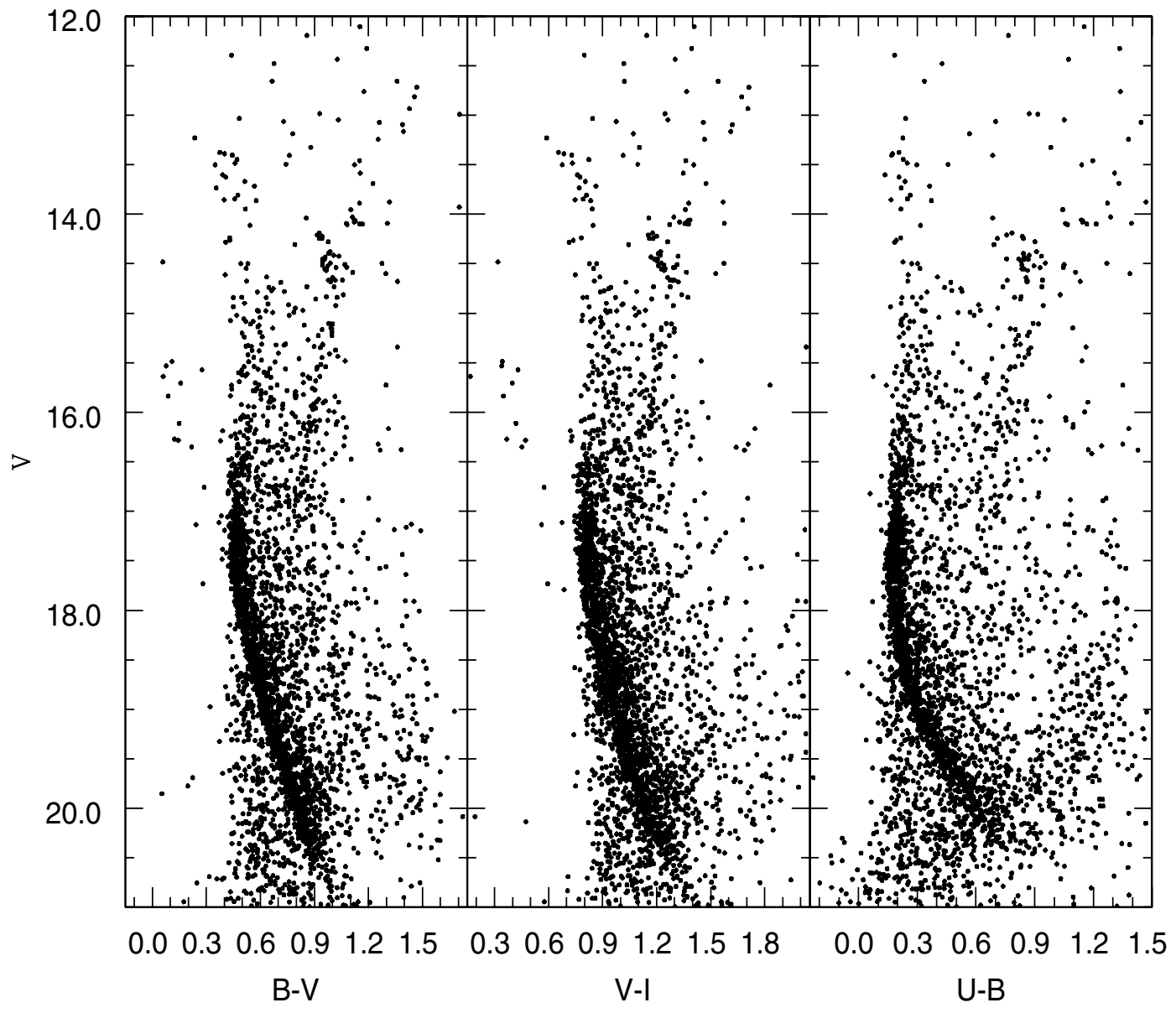

Fig. 5. CMD of Melotte 66. All measured stars are shown.

We make use of the results in the previous section to alleviate the field-star contamination and to have a better handle on the key features of the CMD: the TO, the subgiant branch, the RBG and the clump. With this aim, we select all the stars within the cluster radius, and derive the CMD shown in Fig. 6 in the $B / U-I$ plane. We use this color combination to have a better view of both blue and red parts of the CMD. The MS is very clean and extends down to $B \sim 21.0$. Close to the TO, between $B \sim 17.5$ and $B \sim 18$, the MS broadens, but this broadening is mostly produced by the intersection with the binary sequence, which is well clean down to $B \sim 21$. The cluster TO is then located at $B \sim 18, U-I \sim 1.4$. The subgiant branch and the RGB are more scattered, but the bottom of the RGB is most probably located at $B \sim 17.7, U-I \sim 2.65$. The RGB clump is spread in color, and tilted along the reddening vector, which implies that some differential reddening must be present (Carraro et al. 2002).

To quantify the effect, photometry has been corrected by means of a procedure described in full detail in Milone et al. (2012). Briefly, we iteratively define a fiducial MS for the cluster and then compute the displacement along the reddening vector of each star from this fiducial line. This systematic color and magnitude offset are our estimates of the local differentialreddening values. A map with the resulting reddening variation is shown in Fig. 7. This map indicates that differential reddening is present, and its maximum spatial variation amount to 0.07 mag, which is significantly lower than Zlocewski et al. (2007) estimate, that was based, however, on the low resolution Far InfraRed Background(FIRB) maps (Schlegel et al. 1998). In Fig. 8 we show a zoom of the MS region in Melotte 66 CMD

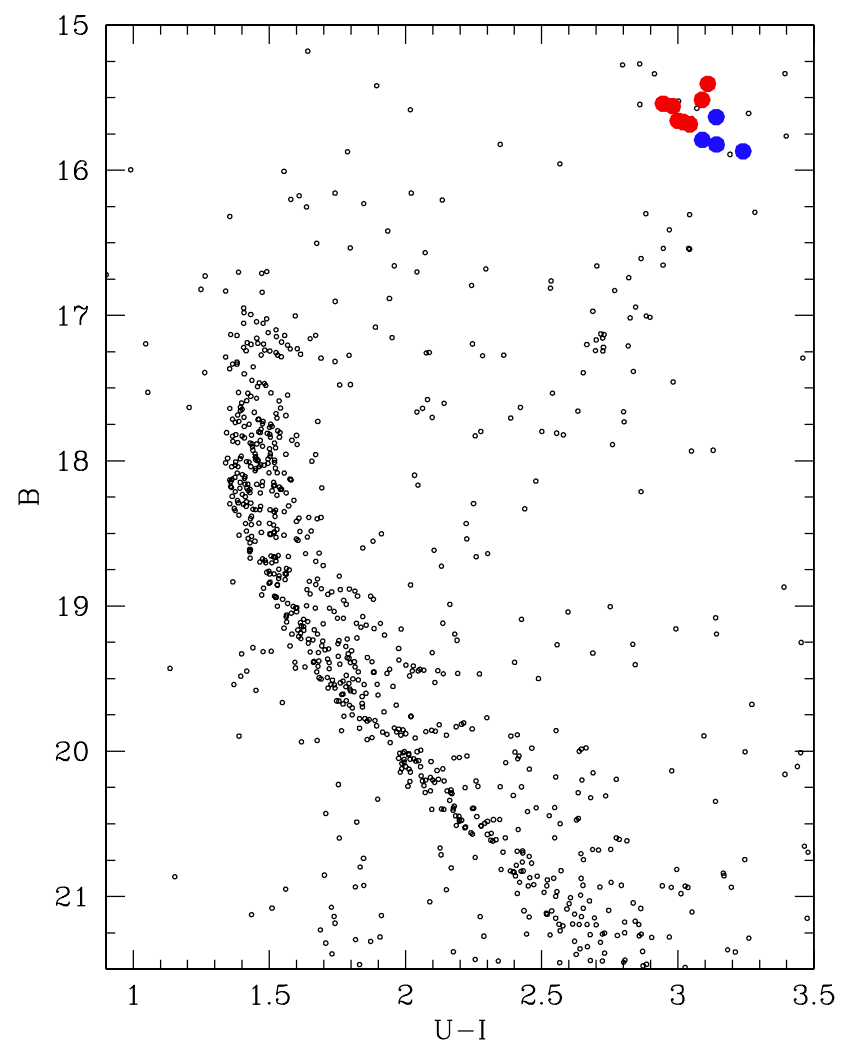

Fig. 6. CMD of Melotte 66. Only stars within 6 arcmin are shown. Clump stars are color-coded: we could obtain high-resolution spectra for the red stars, while the blue are taken from Sestito et al. (2008). 


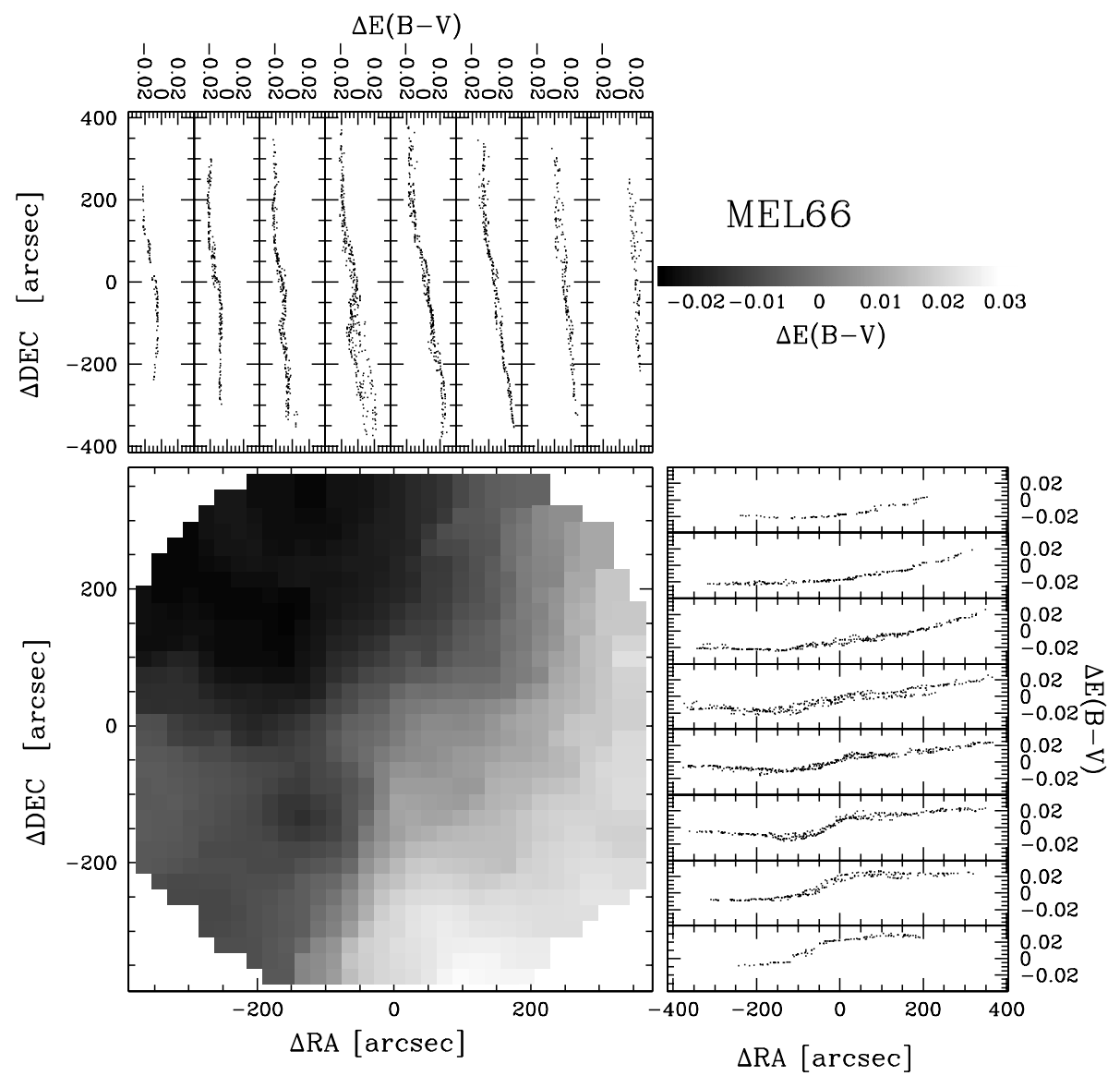

Fig. 7. A reddening map in the region of Melotte 66. See text for details. before and after the variable reddening correction. The corrected MS is clearly less wide than the original, and the overall quality of the CMD improves significantly. Based on these results, the MS broadening can be entirely accounted for by the presence of a certain amount of binary stars and variable extinction across the cluster field. Other scenarios, like extended star formation or metallicity spread, are therefore not required to explain the MS natural width.

To confirm this scenario further, we make use of synthetic CMDs, as generated from the Padova (Bressan et al. 2012) suite of models. The method is described in detail in Carraro et al. (2002). First of all, we need an estimate of the cluster fundamental parameter. With this aim, we make use of isochrone for the metallicity $Z=0.009$, which is derived from the $[\mathrm{Fe} / \mathrm{H}]$ valued obtained in this paper (see below). This is illustrated in Fig. 9, in both the $V / B-V$ and $V / V-I$ plane. The fit is generally good in the MS and TO region. The magnitude of the clump is also well reproduced. There is, however, a clear problem with the color of the RGB, that models predict redder than observations. The best-fit isochrone is for an age of $3.4 \pm 0.2 \mathrm{Gyr}$. The uncertainty in the age has been derived by super imposing many different isochrones and evaluating by eye whether they produced a reasonable fit or not.

For this estimate of the age, we infer a reddening of $E(B-$ $V)=0.15 \pm 0.03$, which is in line with previous estimates, and an apparent distance modulus $(m-M)=13.9 \pm 0.2$. From these two figures we derive an absolute distance modulus $\left(m-M_{0}\right)=13.4 \pm 0.3$. In turn, this is used to estimate an heliocentric distance of $4.7_{-0.1}^{+0.2} \mathrm{kpc}$ for Melotte 66 .

Therefore, Melotte 66 is one of the old open clusters with the largest displacement $(\sim 1.0 \mathrm{kpc})$ from the formal Galactic plane. This, however, has to be considered as an upper limit, since the

\section{ORIGINAL}

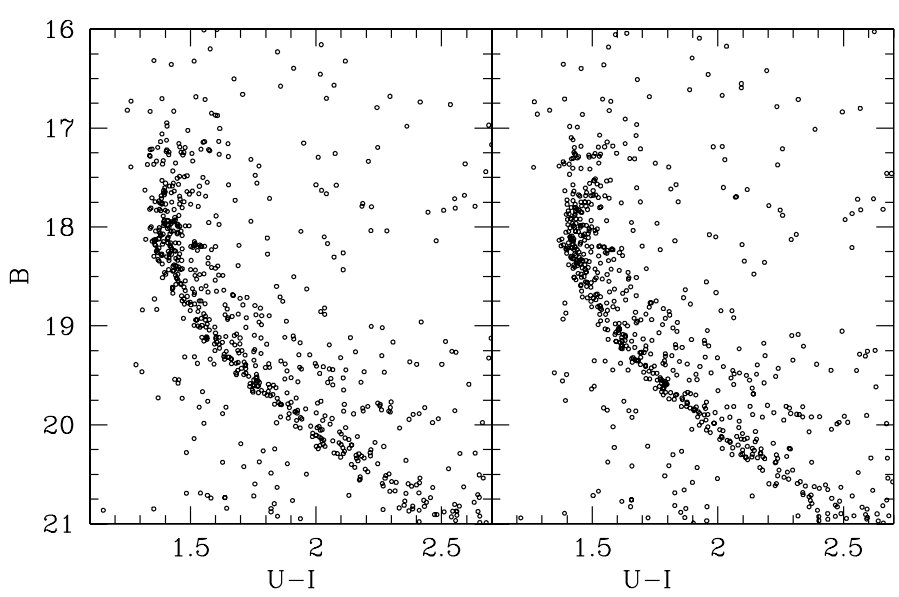

Fig. 8. A zoom of the MS region for Melotte 66 before (left panel) and after (right panel) differential reddening correction.

disk is significantly warped in the cluster direction (Moitinho et al. 2006).

With this information at hand, we generated synthetic CMDs by using the TRILEGAL code (Girardi et al. 2005) and by following Carraro et al. $(2002,2006)$ prescriptions. First of all we generate a synthetic star cluster for the age, reddening, and distance of Melotte 66 , by adding $30 \%$ of binaries with mass ratio in the range 0.6 to 1.0. This is shown in Fig. 10, upper left panel (a). In the upper right panel (b) we show a realization of the expected Milky Way population in the direction of Melotte 66 for an area as large as the one covered by our photometry. The simulation 
Mel 66, $\mathrm{E}(\mathrm{B}-\mathrm{V})=0.15,(\mathrm{~m}-\mathrm{M})=13.9, \tau=3.4 \mathrm{Gyr}$

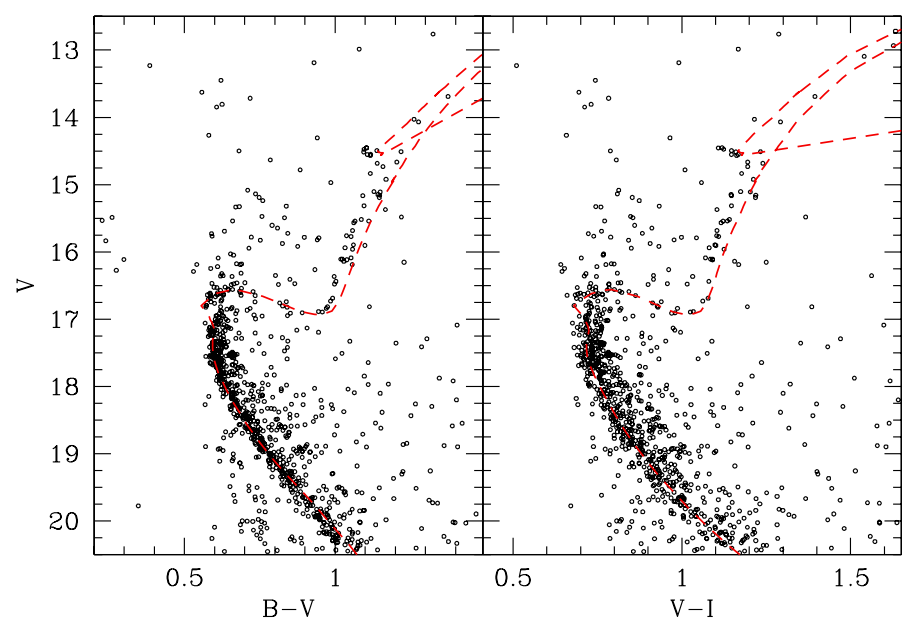

Fig. 9. Isochrone solution for Melotte 66 in the $V / B-V$ (left panel) and $V / V-I$ (right panel) planes. The best fit has been obtained for an age of $3.4 \mathrm{Gyr}, E(B-V)=0.15$, and $(m-M)=13.9$. See text for additional details.

includes stars from the Galactic halo (color-coded in red), and the Galactic thin and thick disk (color-coded in green and blue, respectively). No stars from the Galactic bulge are expected for this specific direction.

In the middle panels ((c) and (d)), we show the same two CMDs blurred by the errors as in our photometric data set. Finally, the lower left panel (e) illustrates the combination of the synthetic cluster plus the synthetic Galactic field, which one has to compare with the real data in the lower right (f) panel.

Overall, the two last panels look very similar, which means that we modeled correctly the field star population statistically speaking, and our ingredients, which may distance, reddening, metallicity and binary properties, are mostly fine. These simulations show that there are many thick disk stars in the line of sight to Melotte 66. They form a sequence which intersect the cluster sub giant branch. Halo and thin disk stars are a minor contribution. It is also evident that field stars do not have colors bluer than the cluster TO, and therefore, there are no field stars in the region of the CMD where blue stragglers are located.

\section{Blue straggler stars in Melotte 66}

As mentioned in Sect. 3, it has been known since the early study from Hawarden (1976) that Melotte 66 harbors a population of blue straggler stars (BSS). According to the most recent compilation by Ahumada \& Lapasset (2007), the cluster hosts as many as 35 BSS, while De Marchi et al. (2006) found 29 BSS within a radius of 7 arcmin from the cluster center. However, as emphasized by Carraro et al. (2008), the precise number of BSS in open clusters is difficult to know because of the severe field star contamination. As a consequence of this, many field stars fall in the region of the $\mathrm{CMD}$, where $\mathrm{BSS}$ are routinely found, which significantly affects their statistics. In the case of Melotte 66, field star contamination is not as important as in many other open clusters because of its relative high Galactic latitude. Still, as shown in Fig 8, some field star contamination is present. This is in the form of a tilted sequence, which crosses the subgiant branch of Melotte 66, and it is mostly composed by thick disk stars (see Sect. 5).

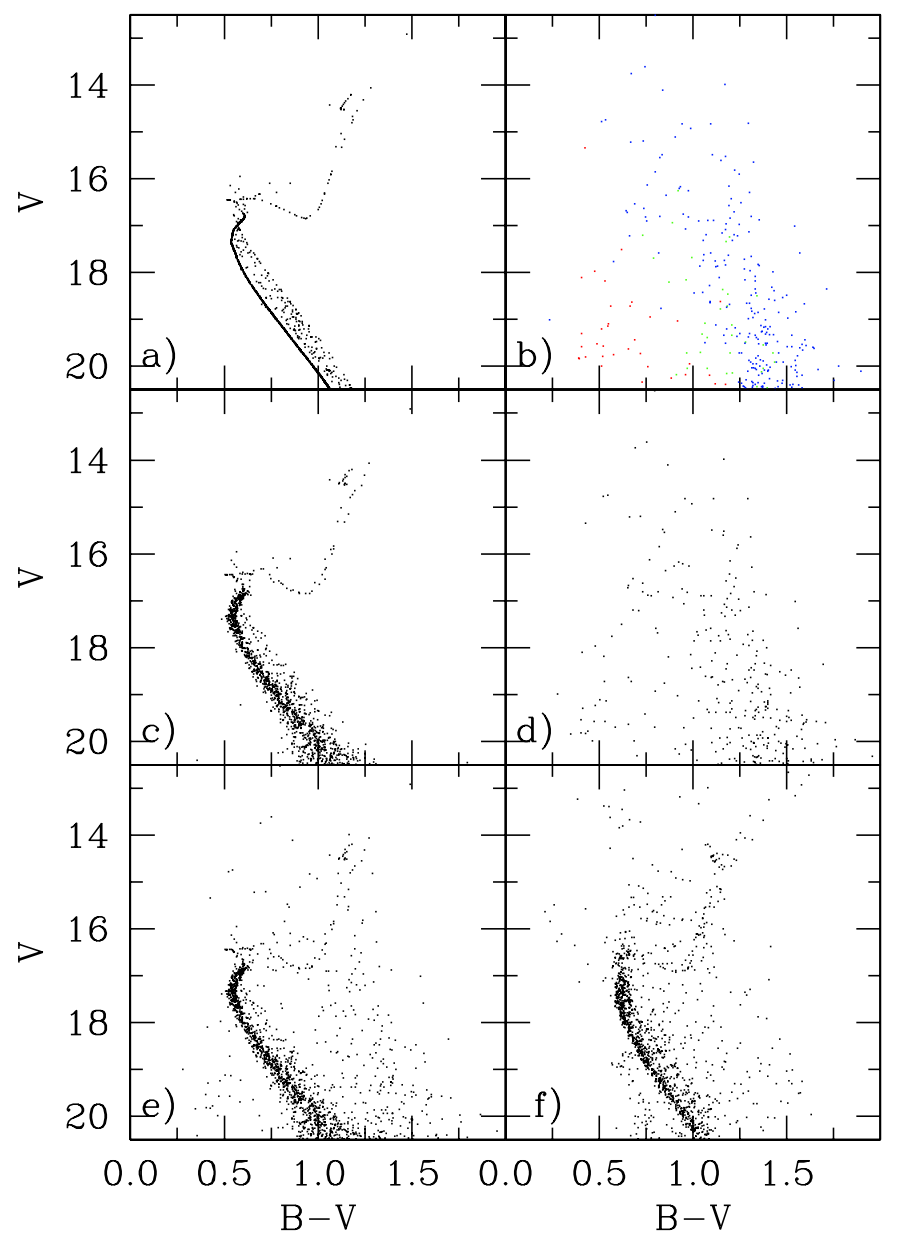

Fig. 10. A simulation of the stellar field in the line of sight toward Melotte 66. See text for more details.

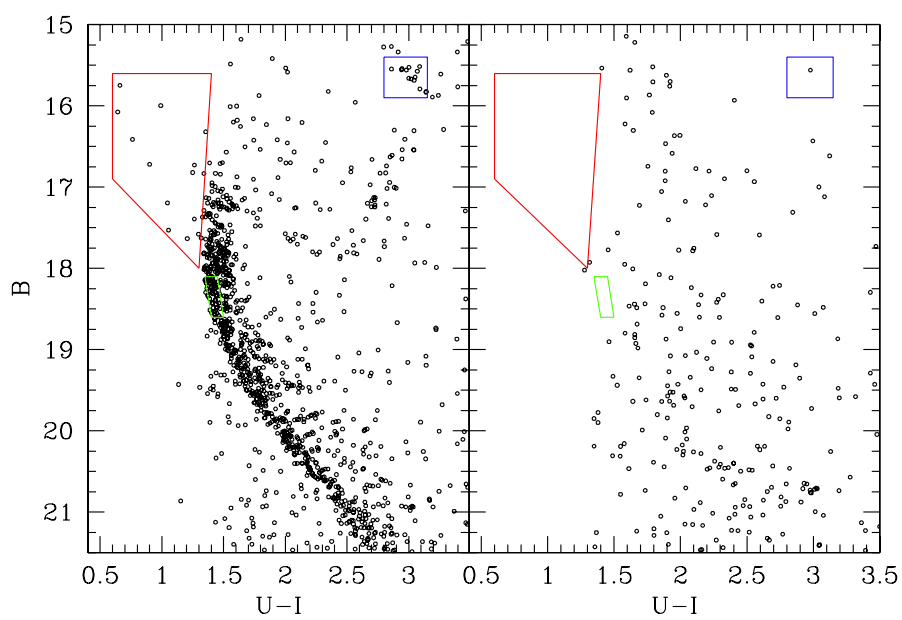

Fig. 11. Selection of BSS, MS, and red-clump stars in Melotte 66 CMD (left panel). An equal area field is shown in the right panel to probe field star contamination.

We start the analysis of the BSS population by selecting BSS candidates in the CMD with a sample of clump and MS stars. This is illustrated in Fig. 13, where the left panel shows the BBS candidate region (red polygon), the clump region (blue square), and a sample of MS single stars (green polygon). In the right panel, an equal area field realization is shown, to see that the selected stars fall in regions that are not significantly contaminated 

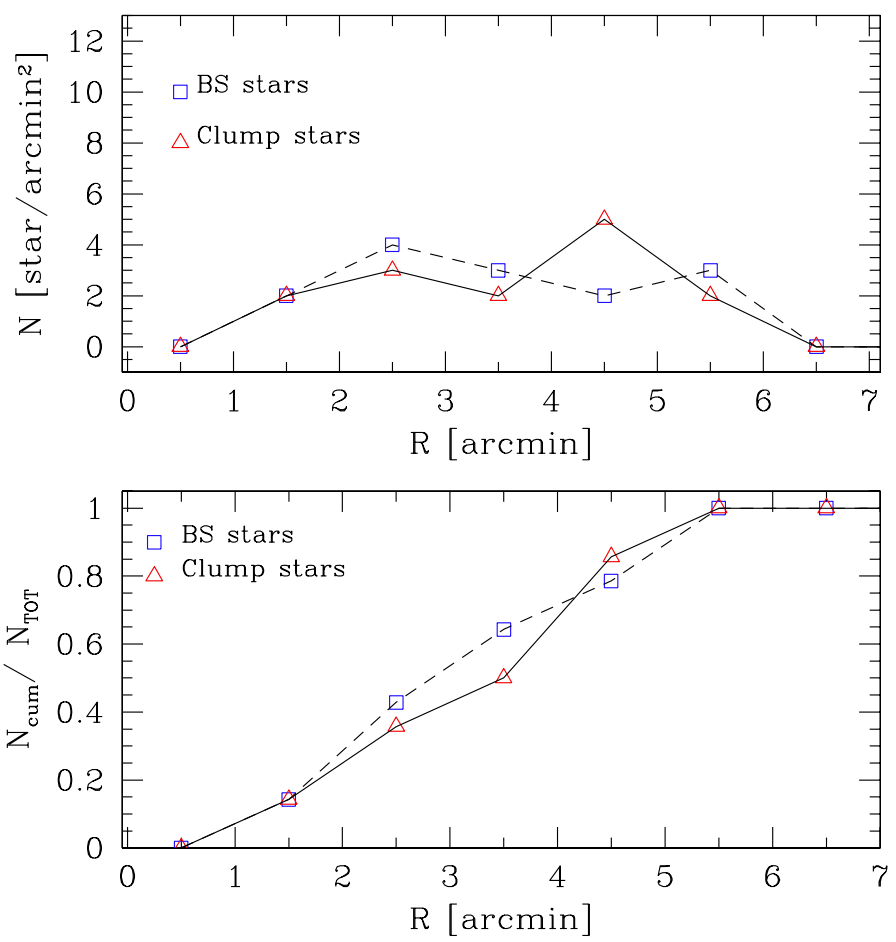

Fig. 12. Upper panel: radial distribution of BSS and clump stars. Lower panel: cumulative radial distribution of BSS and clump stars.
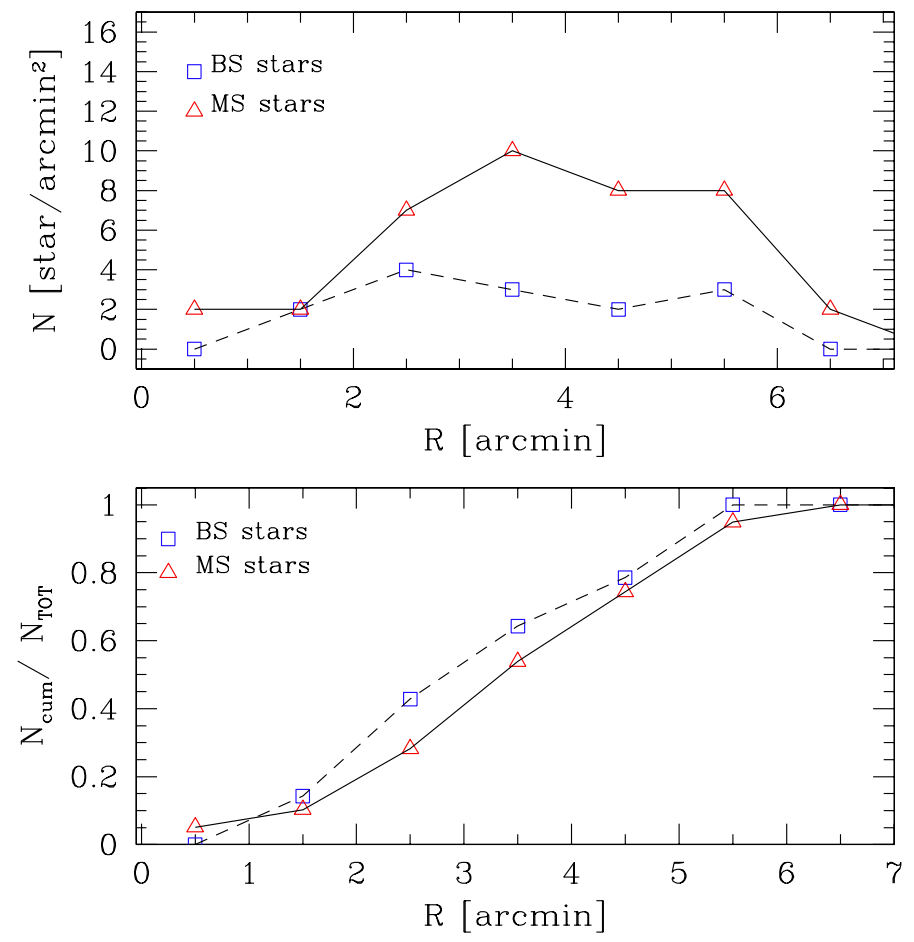

Fig. 13. Upper panel: radial distribution of BSS and MS stars. Lower panel: cumulative radial distribution of BSS and MS stars.

by field stars. MS stars, in particular, have been searched for in a region of the MS not affected by incompleteness and un a region that is most probably free of binary star contamination. As a result, we find 14 candidate BSS and compare them with a sample of bona fide clump stars (14) and MS single stars (39).

The comparison with clump stars is shown in Fig. 14, while the comparison with MS stars is shown in Fig. 15. In both figures, the upper panels present the radial density profile of the two

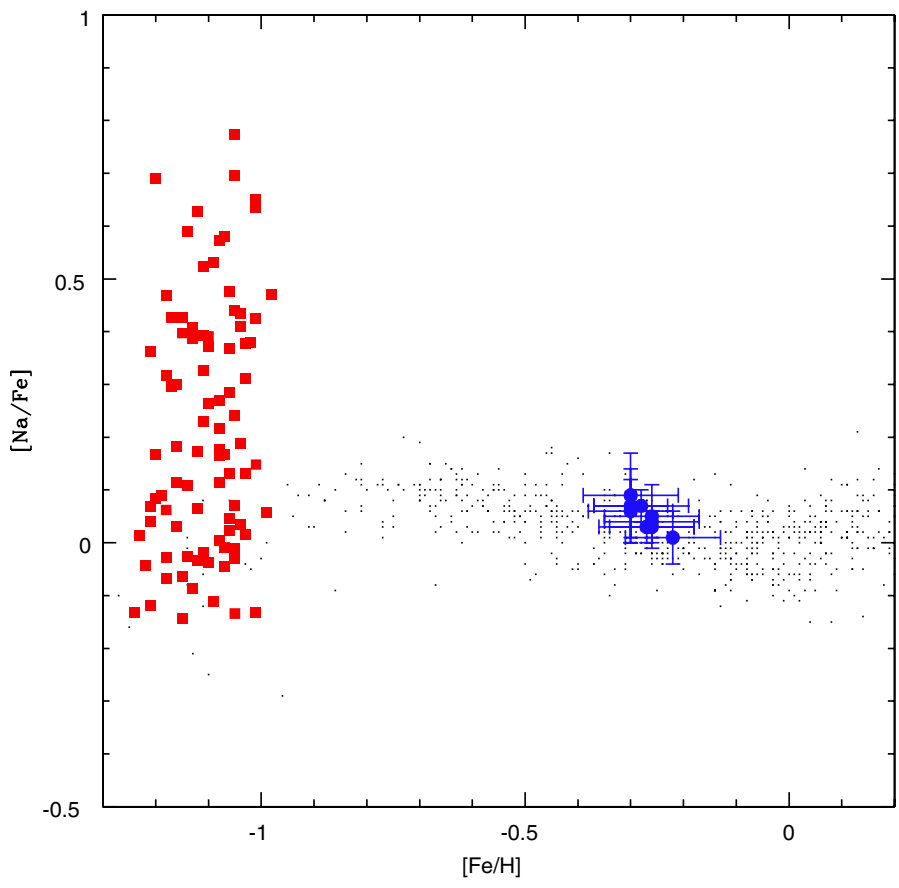

Fig. 14. $[\mathrm{Na} / \mathrm{Fe}]$ vs. $[\mathrm{Fe} / \mathrm{H}]$. The large filled circles are Melotte 66 sample stars. The red squares are NGC 2808 stars from Carretta et al. (2006), and the black points are thin and thick disk stars from Bensby et al. (2014).

populations, while the lower panels present the radial profile of the normalized cumulative distribution (see Carraro \& Seleznev 2011, for more details). Clump stars and BSS do show about the same distribution, and indeed a Kolmogorov-Smirnov (KS) test implies a probability of $79 \%$ when they are drawn from the same parent distribution. There seems to be marginal evidence that BSS are more concentrated than clump stars. A completely different scenario is revealed by comparing MS stars (see Fig. 14). In this case, it seems clear that the BSS are more centrally concentrated than MS single stars, and the KS test in this case returns a $20 \%$ probability that they have the same origin. This analysis lends support to a scenario where BSS stars in Melotte 66 are most probably binaries. The environment of Melotte 66 is quite loose, and therefore they might be primordial binaries that sank toward the cluster center because of their larger combined mass, and then survived in the cluster central regions. A spectroscopic study of these stars would be very useful to support or deny our conclusions.

\section{Observation and data reduction: spectroscopy}

Observations were taken in service mode on the nights of February 12 and March 4, 2012 using the multi-object, fiberfed FLAMES facility mounted at the ESO-VLT/UT2 telescope at the Paranal observatory (Chile). Two 2400s exposures were taken with the red arm of the UVES high-resolution spectrograph setup at $5800 \AA$, a central wavelength that covers the 4760-6840 $\AA$ wavelength range and thus provides a resolution of $R \simeq 47000$.

The data were reduced using the ESO CPL based FLAMESUVES pipeline version 5.0.9 ${ }^{5}$ for extracting the individual fiber spectra.

5 http://www.eso.org/sci/software/pipelines/ 
Table 4. Stellar parameters for all the spectroscopic targets.

\begin{tabular}{lccccccc}
\hline \hline ID & Kassis et al. & Sestito et al. & $\begin{array}{c}T_{\text {eff }} \\
(\mathrm{K})\end{array}$ & $\log g$ & $\begin{array}{c}\xi \\
\mathrm{km} \mathrm{s}^{-1}\end{array}$ & {$[\mathrm{Fe} / \mathrm{H}]$} & $\begin{array}{c}\mathrm{RV} \\
\mathrm{km} \mathrm{s}^{-1}\end{array}$ \\
\hline 597 & 385 & - & 4900 & 1.80 & 1.45 & $-0.30 \pm 0.07$ & $22.63 \pm 0.03$ \\
776 & 603 & - & 4800 & 2.00 & 1.63 & $-0.27 \pm 0.09$ & $21.26 \pm 0.07$ \\
1521 & 1419 & 2218 & 4900 & 2.00 & 1.35 & $-0.30 \pm 0.08$ & $21.29 \pm 0.14$ \\
2099 & 1953 & 1346 & 4850 & 1.90 & 1.37 & $-0.28 \pm 0.09$ & $22.58 \pm 0.65$ \\
2209 & 2155 & - & 4850 & 2.00 & 1.45 & $-0.26 \pm 0.08$ & $22.52 \pm 0.03$ \\
2291 & 2187 & - & 4850 & 1.90 & 1.46 & $-0.26 \pm 0.09$ & $20.13 \pm 0.03$ \\
2803 & 2771 & 1785 & 4850 & 2.00 & 1.38 & $-0.26 \pm 0.09$ & $22.54 \pm 0.14$ \\
1202 & 1000 & 1493 & 4850 & 2.00 & 1.30 & $-0.30 \pm 0.09$ & $22.90 \pm 0.05$ \\
2980 & 2945 & 1865 & 4850 & 2.00 & 1.45 & $-0.32 \pm 0.09$ & $18.76 \pm 0.03$ \\
1919 & 1805 & 1884 & 4850 & 2.50 & 1.30 & $-0.22 \pm 0.09$ & $21.92 \pm 0.03$ \\
\hline
\end{tabular}

Notes. IDs in Col. 1 are from this study.

Table 5. Mean abundances and standard deviations.

\begin{tabular}{|c|c|c|c|c|c|c|c|c|c|c|}
\hline Kassis et al. & Sestito et al. & {$[\mathrm{Na} / \mathrm{Fe}]$} & {$[\mathrm{Mg} / \mathrm{Fe}]$} & {$[\mathrm{Al} / \mathrm{Fe}]$} & {$[\mathrm{Si} / \mathrm{Fe}]$} & {$[\mathrm{Ca} / \mathrm{Fe}]$} & {$[\mathrm{Ti} / \mathrm{Fe}]$} & {$[\mathrm{Cr} / \mathrm{Fe}]$} & {$[\mathrm{Ni} / \mathrm{Fe}]$} & {$[\mathrm{Ba} \mathrm{II} / \mathrm{Fe}]$} \\
\hline 385 & - & $0.07 \pm 0.07$ & $0.19 \pm 0.01$ & $0.18 \pm 0.07$ & $0.05 \pm 0.08$ & $0.10 \pm 0.09$ & $-0.05 \pm 0.07$ & $-0.05 \pm 0.08$ & $-0.08 \pm 0.08$ & $0.35 \pm 0.03$ \\
\hline 603 & - & $0.03 \pm 0.03$ & $0.14 \pm 0.05$ & $0.16 \pm 0.03$ & $0.01 \pm 0.07$ & $0.03 \pm 0.07$ & $-0.08 \pm 0.11$ & $-0.13 \pm 0.06$ & $-0.07 \pm 0.08$ & $0.29 \pm 0.01$ \\
\hline 1419 & 2218 & $0.06 \pm 0.06$ & $0.22 \pm 0.02$ & $0.17 \pm 0.07$ & $0.02 \pm 0.06$ & $0.08 \pm 0.09$ & $-0.09 \pm 0.10$ & $-0.07 \pm 0.06$ & $-0.07 \pm 0.08$ & $0.33 \pm 0.01$ \\
\hline 1953 & 1346 & $0.07 \pm 0.03$ & $0.16 \pm 0.06$ & $0.16 \pm 0.08$ & $0.11 \pm 0.06$ & $0.03 \pm 0.04$ & $-0.10 \pm 0.09$ & $-0.05 \pm 0.07$ & $-0.09 \pm 0.09$ & $0.32 \pm 0.01$ \\
\hline 2155 & - & $0.03 \pm 0.03$ & $0.17 \pm 0.04$ & $0.10 \pm 0.03$ & $0.08 \pm 0.08$ & $0.02 \pm 0.08$ & $-0.07 \pm 0.07$ & $-0.09 \pm 0.07$ & $-0.08 \pm 0.08$ & $0.30 \pm 0.08$ \\
\hline 2187 & - & $0.05 \pm 0.06$ & $0.13 \pm 0.01$ & $0.18 \pm 0.04$ & $0.03 \pm 0.10$ & $0.05 \pm 0.10$ & $-0.08 \pm 0.08$ & $-0.07 \pm 0.05$ & $-0.04 \pm 0.06$ & $0.30 \pm 0.02$ \\
\hline 2771 & 1785 & $0.04 \pm 0.03$ & $0.15 \pm 0.01$ & $0.16 \pm 0.06$ & $0.08 \pm 0.08$ & $0.04 \pm 0.07$ & $-0.06 \pm 0.07$ & $-0.09 \pm 0.10$ & $-0.03 \pm 0.08$ & $0.35 \pm 0.02$ \\
\hline 1000 & 1493 & $0.09 \pm 0.08$ & $0.18 \pm 0.02$ & $0.16 \pm 0.04$ & $0.05 \pm 0.09$ & $0.07 \pm 0.10$ & $-0.10 \pm 0.08$ & $-0.10 \pm 0.07$ & $-0.08 \pm 0.08$ & $0.37 \pm 0.02$ \\
\hline 2945 & 1865 & $0.32 \pm 0.06$ & $0.21 \pm 0.08$ & $0.18 \pm 0.10$ & $0.09 \pm 0.10$ & $0.03 \pm 0.09$ & $-0.10 \pm 0.08$ & $-0.04 \pm 0.08$ & $-0.07 \pm 0.08$ & $0.52 \pm 0.03$ \\
\hline 1805 & 1884 & $0.01 \pm 0.05$ & $0.10 \pm 0.03$ & $0.07 \pm 0.09$ & $-0.02 \pm 0.10$ & $0.01 \pm 0.10$ & $-0.08 \pm 0.09$ & $-0.04 \pm 0.06$ & $-0.07 \pm 0.09$ & $0.26 \pm 0.06$ \\
\hline
\end{tabular}

The spectra were eventually normalized using the standard IRAF task continuum. Radial velocities were computed using the IRAF/fxcor task to cross-correlate the observed spectra with a synthetic one from the Coelho et al. (2005) library with stellar parameters $T_{\text {eff }}=5250 \mathrm{~K}, \log g=2.5$, solar metallicity, and no $\alpha$-enhancement. The IRAF rvcorrect task was used to calculate the correction from geocentric velocities to heliocentric. We took the star's radial velocity to be the average of the two epochs measured and the error to be the difference between the two values multiplied by 0.63 (see Keeping 1962).

Finally, for the abundance analysis, the two epoch restframe spectra obtained for each star were averaged together. The final spectra have signal-to-noise ratios in the range 30-50 at $\sim 6070 \AA$.

\section{High resolution chemical abundances}

\subsection{Abundance analysis}

Our spectroscopic sample consists of seven targets from UVES observations and six targets from Sestito et al. (2008), with six stars in common. The elemental abundances were derived based on equivalent width (EW) measurements using the MOOG abundance calculation code (Sneden 1973). The EWs were measured using the automated ARES code (Sousa et al. 2007) with frequent manual checks of the EWs. Interpolated Kurucz model atmospheres based on the ATLAS9 code (Kurucz 1993; Castelli 1997) with no convective overshooting were used throughout the analysis.

We derive the stellar parameters $\left(T_{\mathrm{eff}}, \log g\right.$, and $\xi$ ) based on spectroscopy. Abundances for all Fe I and II lines were computed from the measured EWs, where we always adopts a starting model of $T_{\text {eff }}=4850 \mathrm{~K}$ and $\log g,=2.5$. The effective temperature was derived by requiring excitation equilibrium of the
Fe I lines. Micro-turbulence was derived from the condition that abundances from Fe I lines show no trend with EWs. Surface gravity was derived via ionization equilibrium, which requires the abundances from Fe I lines to equal those from Fe II lines. The adopted stellar parameters are shown in Table 4 with the measured radial velocity.

Next, we derived elemental abundances for $\mathrm{Na}, \mathrm{Mg}, \mathrm{Al}, \mathrm{Si}$, $\mathrm{Ca}, \mathrm{Ti}, \mathrm{Cr}, \mathrm{Ni}$, and $\mathrm{Ba}$ by adopting the solar abundance values from Grevesse \& Sauval (1998) when calculating the relative abundances. Final abundances are reported in Table 5 along with the corresponding line-to-line standard deviation (rms) from the mean abundances. These results are shown graphically in Fig. 11.

\subsection{Error budget}

Chemical abundances are largely affected by two sources of uncertainties: (i) error in the EW measurement and (ii) error in the stellar parameters $\left(T_{\text {eff }}, \log g\right.$, and $\left.\xi\right)$. There are also uncertainties in the atomic data, known as the $\log g f$, however,m the effect of this is negligible when looking for star-to-star variations given the narrow range of stellar parameters in this sample, where any such effect would systematically affect all stars and not contribute to the random errors internal to this study. The random errors in the abundances due to EW measurements are well represented by the standard deviation (rms) from the mean abundance based on the entire set of lines.

Abundance errors due to stellar parameters were estimated by varying one parameter at a time and checking the corresponding variation in the resulting abundance. We adopted variations of $\pm 50 \mathrm{~K}$ in $T_{\text {eff }}$ and $\pm 0.05 \mathrm{~km}^{-1}$ in $\xi$ because larger changes in those quantities would have introduced a significant trend in $\log n(\mathrm{Fe})$ vs. the excitation potentials and the line strength, 
Table 6. Errors due to stellar parameters.

\begin{tabular}{lcccccccccc}
\hline \hline Parameter & $\mathrm{Fe}$ & $\mathrm{Na}$ & $\mathrm{Mg}$ & $\mathrm{Al}$ & $\mathrm{Si}$ & $\mathrm{Ca}$ & $\mathrm{Ti}$ & $\mathrm{Cr}$ & $\mathrm{Ni}$ & $\mathrm{Ba}$ \\
\hline$\Delta T_{\text {eff }} \pm 50 \mathrm{~K}$ & \pm 0.03 & \pm 0.04 & $\mp 0.03$ & \pm 0.03 & $\mp 0.01$ & \pm 0.05 & \pm 0.07 & \pm 0.06 & \pm 0.02 & \pm 0.01 \\
$\Delta \log g \pm 0.1$ & 0.00 & 0.00 & 0.00 & 0.00 & \pm 0.02 & $\mp 0.01$ & 0.00 & 0.00 & \pm 0.01 & \pm 0.03 \\
$\Delta \xi \pm 0.05$ & $\mp 0.03$ & $\mp 0.01$ & $\mp 0.01$ & 0.00 & $\mp 0.01$ & $\mp 0.03$ & $\mp 0.02$ & $\mp 0.02$ & $\mp 0.03$ & $\mp 0.04$ \\
\hline
\end{tabular}

respectively. The uncertainties in $\log (g)$ were estimated by varying this quantity until the difference between $\log n(\mathrm{FeI})$ and $\log n$ (FeII) is larger than 0.1 dex, when the ionization equilibrium condition is no longer satisfied. The typical error in $\log (g)$ was $0.1 \mathrm{dex}$. The effect of stellar abundances due to stellar parameters are presented in Table 6. Over all, the total error is dominated by the line-to-line scatter with minimal impact from the error in stellar parameters.

\subsection{Literature comparison}

We compare our derived parameters and abundances against those derived by Sestito et al. (2008). We share six stars in common, and overall, the results are consistent within the quoted error budget. A detailed look at the differences are presented in Table 7. For elements heaver than Si only, the quoted average abundances are compared; as for $\mathrm{Na}, \mathrm{Mg}$ and $\mathrm{Al}$, we compared the individual lines common to both studies and the corresponding LTE abundances.

For stellar parameters, our effective temperature values are hotter by no more than $100 \mathrm{~K}$, which is not unreasonable given the various differences in the temperature calculations. For most stars, surface gravity also agrees within expected uncertainties with, perhaps, the exception of star 2218, for which our value is significantly lower than in Sestito et al. (2008). Other notable difference is the $\mathrm{Al}$ abundance, where our values are larger than expected within the quantified uncertainties for stars 1346, 1493 and 1785. It is unclear what the source of this discrepancy is, and the most likely case could be the continuum normalization of the spectra and its effect on the measured EW. Sestito et al. (2008) noted these large line-to-line variations for $\mathrm{Na}, \mathrm{Mg}$, and $\mathrm{Al}$; hence we made the decision to compare abundances per individual line. Despite the different approaches, both studies reach the same conclusion that there are no significant star-tostar abundance variations within the sample.

\subsection{Discussion of abundances}

The abundance analysis of ten red clump stars in Melotte 66 indicates that this sample is very much chemically homogeneous with little abundance scatter within the cluster members. One star (1865) is found to deviate from the cluster mean values for $\mathrm{Na}$ and $\mathrm{Ba}$. This star has a radial velocity outside of the cluster velocity could potentially be a non member. It is also possible that this is a cluster binary star, where mass transfer is triggering an over production of $\mathrm{Na}$ and $\mathrm{Ba}$ (Sneden et al. 2003). Excluding this star, the mean abundances and the standard deviation of nine clump stars in Melotte 66 are given in Table 8.

The abundance patterns of heavy elements observed from stellar photospheres is likely to be a signature of its natal protocluster cloud composition with intrinsic star-to-star variations within a cluster that are expected to be close to zero (De Silva et al. 2006). Our results show that Melotte 66 has little or no intrinsic star-to-star abundance variations, supporting the scenario
Table 7. Comparison with Sestito et al. (2008) for the common stars.

\begin{tabular}{lcccccc}
\hline \hline & 1346 & 1493 & 1785 & 1865 & 1884 & 2218 \\
\hline$\Delta T_{\text {eff }}$ & 100 & 80 & 80 & 133 & 100 & 50 \\
$\Delta \log g$ & 0.1 & 0.15 & 0.05 & 0.05 & 0.05 & 0.39 \\
$\Delta \xi$ & 0.2 & 0.1 & 0.18 & 0.21 & 0.07 & 0.1 \\
$\Delta \mathrm{Na}: 5688$ & 0.06 & 0.08 & 0.00 & -0.02 & 0.19 & -0.01 \\
$\Delta \mathrm{Na}: 6154$ & 0.09 & -0.06 & 0.05 & 0.03 & 0.00 & 0.00 \\
$\Delta \mathrm{Na}: 6160$ & 0.04 & 0.10 & -0.03 & -0.14 & -0.04 & -0.05 \\
$\Delta \mathrm{Mg}: 6318$ & -0.03 & -0.01 & -0.05 & -0.08 & -0.05 & -0.01 \\
$\Delta \mathrm{Al}: 6696$ & 0.30 & 0.31 & 0.07 & 0.07 & 0.16 & 0.09 \\
$\Delta \mathrm{Al}: 6698$ & 0.31 & -0.51 & 0.34 & 0.22 & 0.17 & 0.21 \\
$\Delta[\mathrm{Fe} / \mathrm{H}]$ & -0.09 & -0.05 & -0.04 & -0.02 & -0.08 & -0.01 \\
$\Delta[\mathrm{Si} / \mathrm{Fe}]$ & 0.02 & 0.09 & 0.02 & 0.05 & 0.12 & 0.14 \\
$\Delta[\mathrm{Ca} / \mathrm{Fe}]$ & 0.12 & 0.02 & 0.06 & 0.04 & 0.05 & 0.00 \\
$\Delta[\mathrm{Ti} / \mathrm{Fe}]$ & 0.08 & 0.08 & 0.07 & 0.05 & 0.16 & 0.10 \\
$\Delta[\mathrm{Cr} / \mathrm{Fe}]$ & 0.04 & 0.12 & 0.03 & -0.01 & 0.02 & 0.10 \\
$\Delta[\mathrm{Ni} / \mathrm{Fe}]$ & 0.07 & 0.07 & -0.02 & 0.05 & 0.07 & 0.10 \\
$\Delta[\mathrm{Ba} / \mathrm{Fe}]$ & 0.00 & -0.07 & -0.06 & -0.05 & 0.00 & 0.03 \\
\hline
\end{tabular}

Notes. Only the Sestito star ID is given.

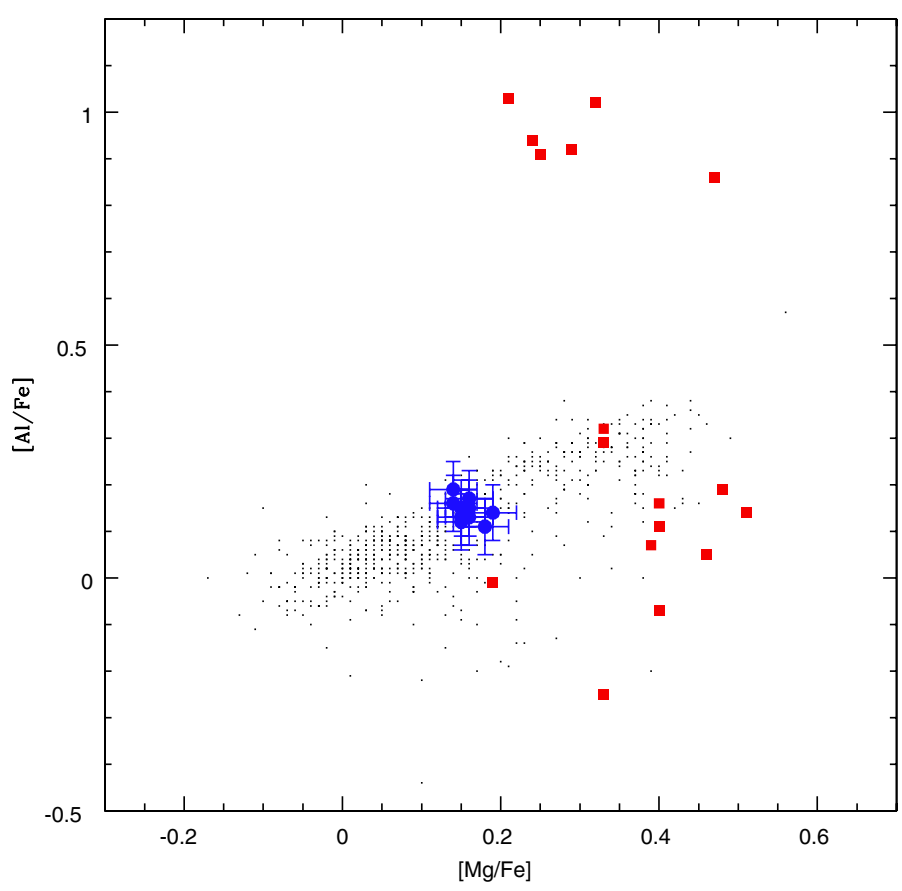

Fig. 15. $[\mathrm{Al} / \mathrm{Fe}]$ vs. $[\mathrm{Mg} / \mathrm{Fe}]$. The large filled circles are Melotte 66 sample stars. The red squares are NGC 2808 stars from Carretta et al. (2006) and the black points are thin and thick disk stars from Bensby et al. (2014).

that it is a typical open cluster in the Galactic disk that is born out of a uniformly mixed proto-cluster gas cloud.

To further explore the apparent lack of multiple stellar populations, we plot $[\mathrm{Na} / \mathrm{Fe}]$ vs. $[\mathrm{Fe} / \mathrm{H}]$ (Fig. 14) and $[\mathrm{Mg} / \mathrm{Fe}]$ vs. [Al/Fe] (Fig. 15) with the abundances of template globular cluster NGC 2808 (red squares from Carretta et al. 2006) and disk 
Table 8. Mean abundances and standard deviation.

\begin{tabular}{ccccccccccc}
\hline \hline & {$[\mathrm{Fe} / \mathrm{H}]$} & {$[\mathrm{Na} / \mathrm{Fe}]$} & {$[\mathrm{Mg} / \mathrm{Fe}]$} & {$[\mathrm{Al} / \mathrm{Fe}]$} & {$[\mathrm{Si} / \mathrm{Fe}]$} & {$[\mathrm{Ca} / \mathrm{Fe}]$} & {$[\mathrm{Ti} / \mathrm{Fe}]$} & {$[\mathrm{Cr} / \mathrm{Fe}]$} & {$[\mathrm{Ni} / \mathrm{Fe}]$} & {$[\mathrm{Ba} \mathrm{II} / \mathrm{Fe}]$} \\
\hline Mean & -0.27 & 0.05 & 0.16 & 0.15 & 0.05 & 0.05 & -0.08 & -0.07 & -0.07 & 0.32 \\
std & 0.03 & 0.03 & 0.04 & 0.04 & 0.04 & 0.03 & 0.02 & 0.03 & 0.02 & 0.03 \\
\hline
\end{tabular}

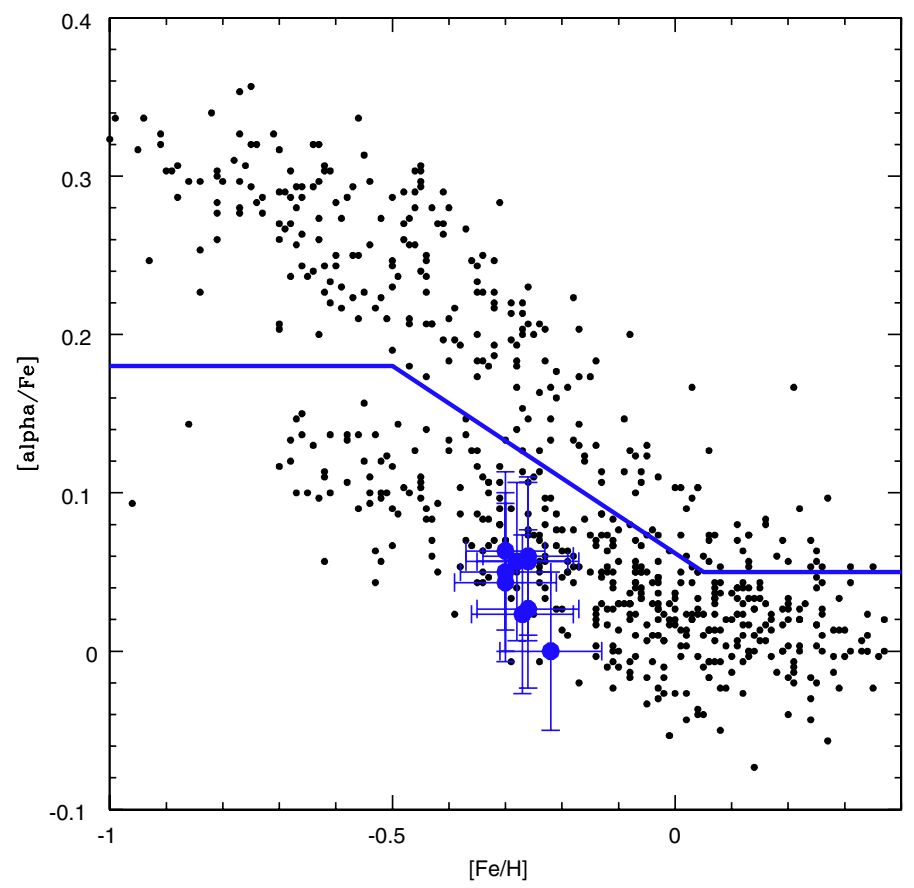

Fig. 16. $[\alpha / \mathrm{Fe}]$ vs. $[\mathrm{Fe} / \mathrm{H}]$ for Melotte 66 sample stars (blue circles). The black points are thin and thick disk star from Bensby et al. (2014), and the line dividing the thin and thick disk is from Haywood et al. (2013).

field stars (black points, from Bensby et al. 2014). The plots shows very clearly that Melotte 66 abundances do not reach the extreme chemical enhancements that indicate multiple stellar populations in globular clusters.

We now compare the abundances of Melotte 66 with other clusters studied with high resolution spectroscopy. Figure 18 shows the average Melotte 66 abundances from this study against other open cluster average abundances from Pancino et al. (2010). The cluster sits within the general spread of the open cluster ratios, and no unusual variations are seen.

We now explore the cluster abundances against the characteristic abundances of the thin and thick disk to better understand the cluster origin. In Fig. 16, we plot the mean $[\alpha / \mathrm{Fe}]$ against $[\mathrm{Fe} / \mathrm{H}]$ for the cluster and Bensby et al. (2014), where $\alpha$ elements represent the average abundances of $\mathrm{Mg}, \mathrm{Si}$, and $\mathrm{Ti}$. The plot also shows the suggested thin-thick disk division line by Haywood et al. (2013) to better guide the eye. It is clear that Melotte 66 sits within the general thin disk population rather than the thick disk.

Our abundance results show that the alpha elements $\mathrm{Si}, \mathrm{Ca}$, and $\mathrm{Na}$ are close to solar ratios in Melotte 66, while $\mathrm{Mg}$ and $\mathrm{Al}$ are enhanced by 0.15 dex relative to $\mathrm{Fe}$. It is also clear that $\mathrm{Ba}$, the only neutron capture element, is enhanced in this cluster. All these elements are, however, within the trend described by disk field stars.

The remaining elements, $\mathrm{Ti}, \mathrm{Cr}$, and $\mathrm{Ni}$ are consistently under abundant relative to Fe by about $\sim 0.07$ dex. This was also seen in the Sestito et al. (2008) study, suggesting that this is a feature of the chemical abundance pattern of Melotte 66. In

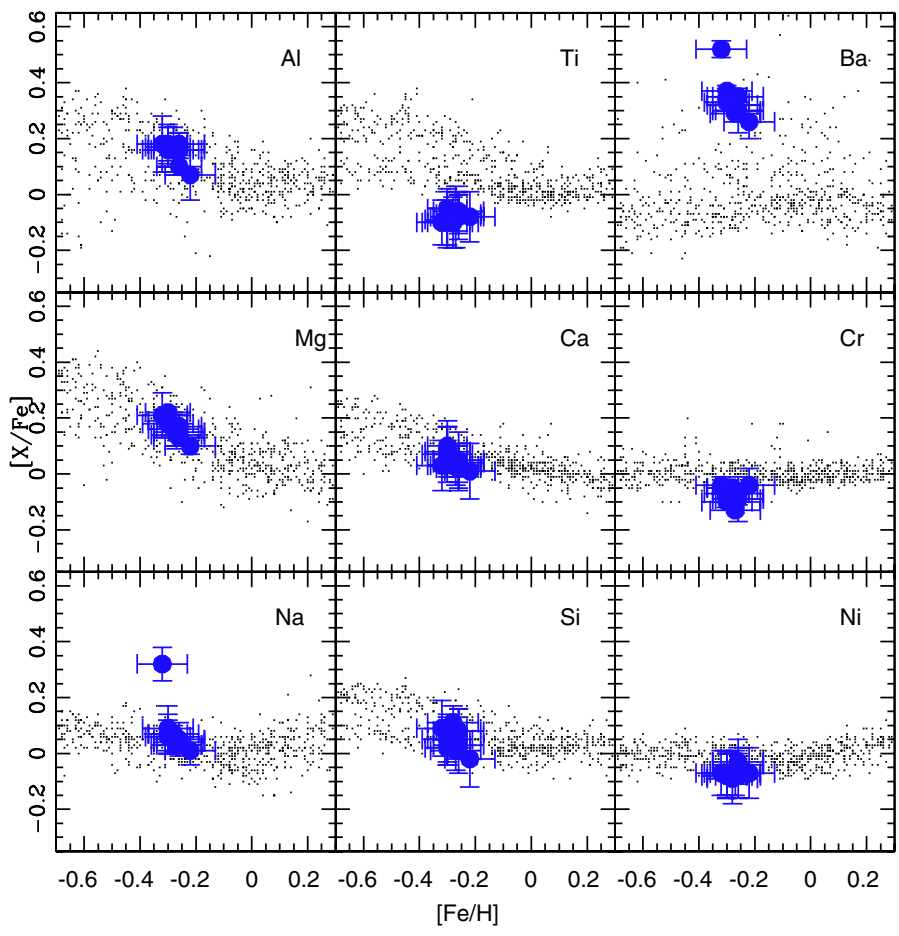

Fig. 17. Abundance ratios in the form of $[X / \mathrm{Fe}]$ vs. $[\mathrm{Fe} / \mathrm{H}]$. The large filled circles are Melotte 66 sample stars. The black points are thin and thick disk stars from Bensby et al. (2014).

Fig. 17, it is clear that the cluster stars sit below the bulk of the thin disk stars for $\mathrm{Ti}$ and also marginally for $\mathrm{Cr}$ and Ni. Similar abundance patterns where Ti behaves more like an Fe-peak element, rather than an alpha-capture element have been observed among other clusters as well (e.g. NGC 2324 and NGC 2477, Bragaglia et al. 2008). Ti remains an intriguing element with its nucleosynthesis origins that are not fully understood.

While a given open cluster is highly chemically homogeneous internally, this highlights abundance variations are seen among the open cluster population (see also De Silva et al. 2009). The likely explanation for such different abundance patterns across the open clusters is due to the different starformation and chemical feedback histories for different regions of the Galaxy. Such differences are key to chemically identifying stars that formed together during the build up of the Galaxy, which are now dissolved into a the general field population (Mitschang et al. 2014).

\section{Conclusions}

We have presented in this paper a photometric and spectroscopic study of Melotte 66, one of the most massive old open clusters in the Milky Way disk. The most important result of our investigation is that Melotte 66 does not show any evidence, either photometric or spectroscopic, of distinct sub populations among its stars. Our photometry demonstrates beyond any reasonable doubt that the MS width is produced by the presence of a significant population of binary stars. The binary sequence intersects 


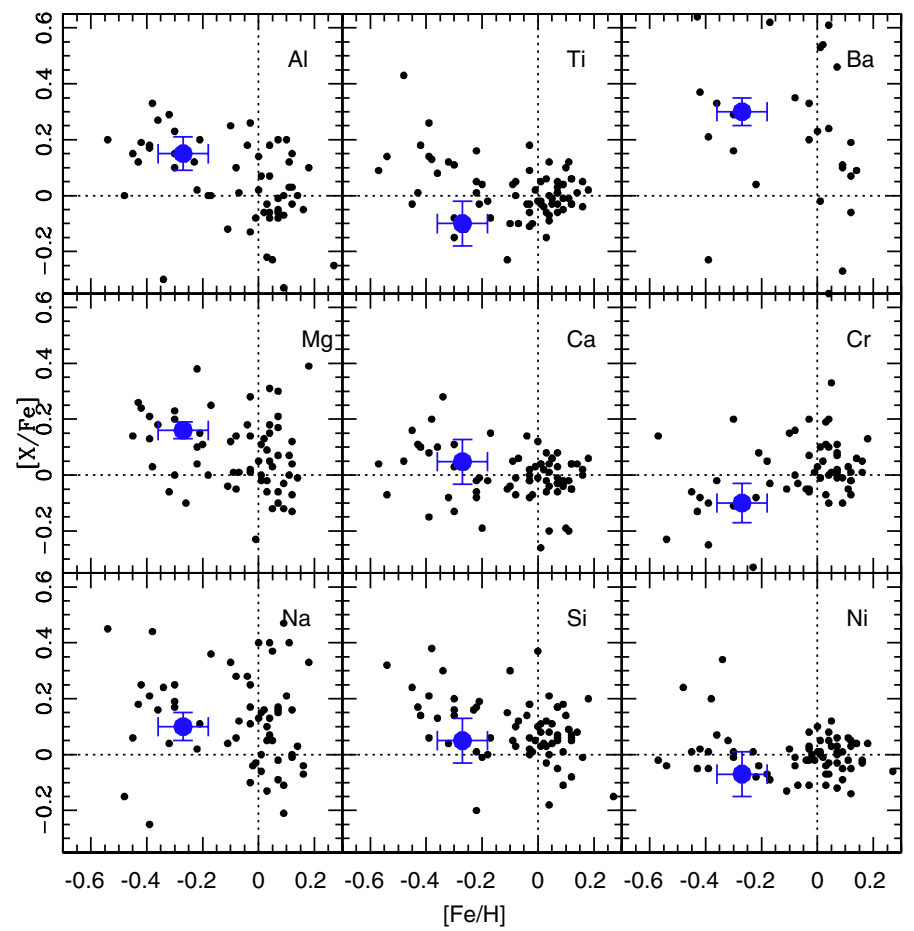

Fig. 18. Average abundance ratios of Melotte 66 (blue circle) compared to literature cluster values from Pancino et al. (2010). The cross-hairs highlight the solar abundances.

the single star MS close to the TO, producing the visual effect that the MS is wide. For the first time, using numerical simulations, we quantify the binary fraction, which would be not smaller than $30 \%$.

We discussed the cluster photometric properties and revised its fundamental parameters. The age is found to be $3.4 \pm 0.2 \mathrm{Gyr}$, which is younger than in previous investigations.

The new spectroscopic material we add fully supports the conclusions from photometry. While confirming previous determinations of $[\mathrm{Fe} / \mathrm{H}]$, we did not detect any significant spread in any of the elements we could analyze. Melotte 66 looks like a genuine member of the old, thin disc population when compared with other disc open clusters and disc field stars.

We finally perform a photometric study of the BSS population in the cluster. We found 14 BBS candidates, a value that is fewer than that found in previous studies, and which we suggest to be primordial binaries.

In conclusion, Melotte 66, like Berkeley 39 (Bragaglia et al. 2012), is a single population star cluster. Although limited by the small number statistics, NGC 6791 seems to be the only open cluster with evidence of multiple stellar populations. The reader, however, has to be warned that NGC 6791 is questioned as a disc star cluster (Carraro 2013; Carrera 2012), since its properties are closer to the bulge stellar population.

Besides Melotte 66 and Berkeley 39, there are not many massive old open cluster candidates. With caution in the introduction to this paper we had described that masses for these clusters are extremely uncertain, and one can possibly look at Collinder 261 or Trumpler 5 as possible targets for further studies in this direction.
Acknowledgements. G. Carraro acknowledges financial support from the Anglo Australian Observatory Distinguished Visitor Program and from the ESO Director General Discretionary Funds, that allowed a visit to AAO In February 2014, where most of this work was done. We warmly thank Russell Cannon and Sarah Martell for useful comments.

\section{References}

Ahumada, J. A., \& Lapasset, E. 2007, A\&A, 463, 789

Anthony-Twarog, B. J., Twarog, B. A., \& McClure, R. D. 1979, ApJ, 233, 188

Anthony-Twarog, B. J., Twarog, B. A., \& Sheeran, M. 1994, PASP, 106, 499

Bensby, T., Feltzing, S., \& Oey, M. S. 2014, A\&A, 562, A71

Bragaglia, A., Sestito, P., Villanova, S., et al. 2008, A\&A, 480, 79

Bragaglia, A., Gratton, R., Carretta, E., et al. 2012, A\&A, 548, A122

Bressan, A., Marigo, P., Girardi, L., et al. 2012, MNRAS, 427, 127

Carraro, G. 2013, Proc. 10th Pacific Rim Conference on Stellar Astrophysics, ASP Conf. Ser., in press [arXiv: 1308.5195]

Carraro, G., \& Seleznev, A. F. 2011, MNRAS, 412, 1361

Carraro, G., Girardi, L., \& Marigo, P. 2002, MNRAS, 332, 705

Carraro, G., Subramaniam, A., \& Janes, K. A. 2006, MNRAS, 371, 1301

Carraro, G., Vázquez, R. A., \& Moitinho, A. 2008, A\&A, 482, 777

Carrera, R. 2012, ApJ, 758, 110

Carretta, E., Bragaglia, A., \& Gratton, R. G., 2006, A\&A, 450, 523

Carretta, E., Bragaglia, A., Gratton, R., et al. 2014, A\&A, 561, A87

Castelli F., Gratton R. G., \& Kurucz R. L. 1997, A\&A, 318, 841

Coelho, P., Barbuy, B., Meléndez, J., Schiavon, R. P., \& Castilho, B. V. 2005, A\&A, 443, 735

Crawford, D. L. 1958, ApJ, 128, 185

Dawson, D. W. 1978, AJ, 83, 1424

De Marchi, F., De Angeli, F., Piotto, G., Carraro, G., \& Davies, M. B. 2006, A\&A, 459, 489

Friel, E. D., \& Janes, K. A. 1993, A\&A, 267, 75

Friel, E. D., Janes, K. A., Tavarez, M., et al. 2002, AJ, 124, 2693

Friel, E. D. 1995, ARA\&A, 33, 381

Geisler, D., Villanova S., Carraro, G., et al. 2012, ApJ, 756, L40

Girardi, L., Groenewegen, M. A. T., Hatziminaoglou, E., \& da Costa, L. 2005, A\&A, 436, 895

Gratton, R., \& Contarini, G. 1994, A\&A, 283, 911

Grevesse, N., \& Sauval, A. J. 1998, Space Sci. Rev., 85, 161

Hamuy, M., Folatelli, G., Morrell, N. I., et al. 2006, PASP, 118, 2

Hawarden, T. G. 1976, MNRAS, 174, 471

Hawarden, T. G. 1978, MNRAS, 182, 31

Haywood, M., Di Matteo, P., Lehnert, M. D., Katz, D., \& Gómez, A. 2013, A\&A, 560, A109

Hogg, A. R. 1965, Mem, Mt. Stromlo Obs., 17

Landolt, A. U. 1992, AJ, 104, 340

Kassis, M., Janes, K. A., Friel, E. D., \& Phelps, R. A. 1997, AJ, 113, 1723

Keeping, E. S. 1962, Introduction to Statistical Inference (Princeton: van Nostrand)

King, I. 1964, R. Obs. Bull., 82, 106

Kurucz, R. L. 1993, CD-ROM 13, 18, http://kurucz .harvard.edu

Mashonkina, L. I., Vinogradova, A. B., Ptitsyn, D. A., Khokhlova, V. S., \& Chernetsova, T. A. 2007, Astron. Rep., 51, 903

Milone, A., Bedin, L. R., Piotto, G., \& Anderson, J. 2009, A\&A, 497, 755

Milone, A. P., Piotto, G., Bedin, L. R., et al. 2012, A\&A, 540, A16

Mitschang, A. W., De Silva, G., Zucker, D. B., et al. 2014, MNRAS, 438, 2753

Moitinho, A., Vázquez, R. A., Carraro, G., et al. 2006, MNRAS, 368, L77

Patat, F., \& Carraro, G. 2001, MNRAS, 325, 1591

Schlegel, D. J., Finkbeiner, D. P., \& Davis, M. 1998, ApJ, 500, 525

Sestito, P., Bragaglia, A., Randich, S., et al. 2008, A\&A, 488, 843

Sneden, C. A. 1973, Ph.D. Thesis, the University of Texas at Austin

Sneden, C. A., Preston, G. W., \& Cowan, J. J. 2003, ApJ, 592, 504

Sousa, S. G., Santos, N. C., Israelian, G., Mayor, M., \& Monteiro, M. J. P. F. G. 2007, A\&A, 469, 783

Stetson, P. B. 1987, PASP, 99, 191

Villanova, S., Geisler, D., Carraro, G., Moni Bidin, C., \& Munoz, C. 2013, ApJ, 778,186

Zloczewski, K., Kaluzny, J., Krzemisnki, W., Olech, A., \& Thompson, I. B. 2007, MNRAS, 380, 1191 